\title{
Étude des restes humains de Kindoki (République démocratique du Congo, fin $\mathrm{XVII}^{\mathrm{e}}-$ début $\mathrm{XIX}^{\mathrm{e}}$ siècle)
}

\author{
Study of the Human Remains from Kindoki (Democratic Republic of Congo, End 17th-Early \\ 19th Century AD)
}

\author{
C. Polet $\cdot$ B. Clist $\cdot$ K. Bostoen \\ Reçu le 2 mai 2017; accepté le 11 septembre 2017 \\ C Société d'Anthropologie de Paris et Lavoisier SAS 2017
}

\begin{abstract}
Résumé Nous présentons l'étude anthropologique de dix individus provenant d'un cimetière situé sur la colline de Kindoki au Kongo central (RDC). Ils ont été exhumés en 2012 et 2013 dans le cadre du projet KongoKing qui consistait en une approche interdisciplinaire de l'histoire ancienne du royaume Kongo. Ce cimetière contient des inhumations datées $\mathrm{du} \mathrm{XVII}^{\mathrm{e}}$ au XIX ${ }^{\mathrm{e}}$ siècle, très probablement celles de nobles de haut rang. Les défunts étaient accompagnés d'un mobilier funéraire (perles de verre, sabres, bijoux en or, mousquet). Les restes humains sont malheureusement très mal conservés. Certains ne sont représentés que par quelques fragments osseux ou dentaires. Parmi les dix défunts, on note la présence de deux femmes et de huit hommes. Deux individus masculins présentent des usures dentaires qui résulteraient de l'utilisation d'une pipe. Les deux femmes seraient âgées de plus de 40 ans et se caractérisent par des signes vertébraux évocateurs de DISH (diffuse idiopathic skeletal hyperostosis). Cette pathologie est actuellement associée à une alimentation riche, à l'obésité et au diabète de type II. Il n'est donc pas surprenant qu'elle touche ces individus de statut élevé. Le DISH n'avait encore jamais été décrit dans une population ancienne d'Afrique centrale.
\end{abstract}

Mots clés République démocratique du Congo ·

Archéologie historique - Époque contemporaine · Royaume Kongo $\cdot$ Statut social

Abstract This paper presents an anthropological study of ten individuals from a cemetery situated on Kindoki Hill in the

\footnotetext{
C. Polet $(\square)$

Institut royal des Sciences naturelles de Belgique,

29, rue Vautier, B-1000 Bruxelles, Belgique

e-mail : caroline.polet@sciencesnaturelles.be

B. Clist $\cdot$ K. Bostoen

BantUGent - Centre d'études bantoues de l'UGent,

Département de Langues et Cultures, Université de Gand
}

central Kongo region (DRC). They were excavated in 2012 and 2013 as part of the KongoKing project (2012-2016), which applied an interdisciplinary approach to the ancient history of the Kingdom of Kongo. The cemetery contains graves dated to the 17th-19th century, very probably those of high-ranking nobles. The dead were buried with various grave goods (glass beads, swords, gold jewels and muskets). The human remains were unfortunately very badly preserved. Some were represented only by a few bone fragments and/or teeth. Of the ten bodies, two were female and eight were male. Two of the men showed dental wear as a result of pipe-smoking. The two women were probably more than 40 years of age at the time of death. The vertebrae showed signs that evoke diffuse idiopathic skeletal hyperostosis (DISH), a pathological condition associated today with a rich diet, obesity and type II diabetes. It is therefore not surprising that these conditions might have affected high-status individuals. Until now, DISH had never been described in an ancient Central African population.

Keywords Democratic Republic of Congo · Modern period · Historical archaeology $\cdot$ Kingdom of Kongo · Social status

\section{Introduction}

Cette étude fait partie du projet interdisciplinaire KongoKing (2012-2016) qui visait à mieux connaître l'origine et l'histoire du royaume Kongo, principalement d'un point de vue politique, linguistique et économique [1]. Ce projet réunissait six institutions : l'Université de Gand, l'Université libre de Bruxelles et le Musée royal d'Afrique centrale, du côté belge ; l'Université de Kinshasa, l'Université Kongo et l'Institut des Musées Nationaux du Congo, du côté congolais. L'Institut royal des Sciences naturelles de Belgique (IRSNB) 
a été contacté en cours de projet pour réaliser l'étude anthropologique des restes humains exhumés.

Partant du constat que les recherches sur l'urbanisation en Afrique centrale ont jusqu'à présent suscité peu d'intérêt, les chercheurs du projet KongoKing ont mis en commun leurs potentiels pour tenter de combler une partie de ces lacunes [2]. Leur choix s'est porté sur le royaume Kongo qui figure parmi les rares structures politiques précoloniales de l'Afrique centrale à être historiquement documentées dès le début du XVI ${ }^{\mathrm{e}}$ siècle. Ce royaume, fondé au XIV ${ }^{\mathrm{e}}$ siècle, occupait un territoire qui est actuellement celui de l'Angola et de la République démocratique du Congo (RDC) [3]. Si l'on excepte les cités historiques de Mbanza Kongo, de Mbanza Soyo et de Ngongo Mbata qui ont fait anciennement l'objet de fouilles archéologiques [4-7], l'histoire des anciennes agglomérations kongo demeure assez méconnue.

Les recherches archéologiques de ce projet se sont concentrées depuis 2012 sur le bassin de l'Inkisi, l'un des principaux affluents du fleuve Congo dans la province du Kongo central (appelée Bas-Congo jusqu'en 2015). Les capitales des provinces de Mbata, de Nsundi et de Mpangu du royaume Kongo se sont en effet établies dans cette vallée $[2,3]$.

Cet article présente les premiers résultats de l'étude des restes humains exhumés lors de ces campagnes de fouilles. Elle porte sur les squelettes découverts sur la colline de Kindoki.

\section{Matériel}

La colline de Kindoki (05 04'069” S ; 1501'403” E) est actuellement située en RDC, plus particulièrement dans la province du Kongo central (Fig. 1AB). Elle s'étend sur une superficie d'environ 30 ha. Son substrat géologique est composé de schiste calcaire de l'unité Bangu. Son sous-sol contient des sables qui sont plus ou moins argileux, avec souvent des galets à la base [8]. La province du Kongo central est caractérisée par un climat tropical humide (selon le système de classification de Köppen, elle appartient au type climatique $\mathrm{Aw}_{4}$ ) [9]. La saison pluvieuse alterne avec la saison sèche. La température moyenne annuelle oscille autour de $25^{\circ} \mathrm{C}$. La moyenne annuelle des précipitations s'élève à environ $1600 \mathrm{~mm}$. L'humidité relative sur l'ensemble de la province se situe autour de $80 \%$ pour tous les mois de l'année [10].

Au temps du royaume Kongo, la colline était située dans la province de Nsundi (Fig. 1C), une zone probablement densément peuplée avec des sols relativement fertiles et une végétation mixte constituée de savanes et de forêts [11]. Depuis le $\mathrm{xvI}^{\mathrm{e}}$ siècle, elle faisait vraisemblablement partie de l'ancienne capitale de cette province (Mbanza Nsundi) [2,3].
L'équipe du projet KongoKing a réalisé des fouilles en 2012, en 2013 et en 2015 sur le site de Kindoki [2]. Les fouilles ont été installées sur un axe est-ouest de $700 \mathrm{~m}$ et sur un axe nord-sud de $450 \mathrm{~m}$. Elles ont combiné le creusement de tranchées tests systématiques et des carroyages à grande échelle. Un cimetière fut ainsi découvert et fit l'objet d'une fouille exhaustive sur une superficie d'environ $279 \mathrm{~m}^{2}$. Des zones d'habitat datées du XIII ${ }^{\mathrm{e}}$ au $\mathrm{xIx}^{\mathrm{e}}$ siècle ont également été mises au jour à proximité du cimetière.

Ce cimetière contenait 11 sépultures qui ont été numérotées $1,2,4,5,6,7,8,9,11,12$ et 13 (Fig. 2). Elles présentaient toutes la même orientation (SW-NE), étaient très proches les unes des autres et avaient été édifiées de manière identique [2]. Une fois le corps déposé, avec son matériel funéraire à plus ou moins $2 \mathrm{~m}$ de profondeur, la fosse était entièrement rebouchée; puis on installait une série de petites dalles matérialisant le rectangle de la fosse. À l'intérieur de cet espace, on plaçait ensuite un pavement de dalles plus grandes. Pour finir, on empilait sur le pavement des pierres irrégulières pour former un cairn. Ces données suggèrent que ce cimetière a été constitué dans un faible laps de temps et que les individus inhumés étaient liés les uns aux autres d'une manière qui reste à identifier.

Quelques indices archéologiques et pédologiques témoignent du traitement des défunts. Dans les sédiments des tombes 1,8 et 11, la présence d'une zone ovalaire de couleur foncée entourant les restes humains suggère la décomposition de couches de tissus emballant le corps (des restes textiles ont d'ailleurs été découverts dans la sépulture 8) (Tableau 1). Cette pratique est attestée par des textes datant $\mathrm{du}$ début $\mathrm{du} \mathrm{xx}^{\mathrm{e}}$ siècle, qui mentionnent que les personnages de statut social élevé étaient emballés dans des dizaines, voire des centaines de couches de tissus (tapis importés, nattes, etc.) [2,3]. La tombe 4 a livré des clous en fer entourant le défunt, témoins d'un cercueil en bois. Les sept autres tombes n'ont livré aucune structuration particulière, laissant penser à un dépôt simple des corps en pleine terre ou à un appareil funéraire dont les traces n'étaient plus perceptibles au moment de la fouille.

Les défunts étaient accompagnés d'un mobilier funéraire dont la composition varie d'une sépulture à l'autre (les tombes 1 et 13 en étaient toutefois dépourvues) (Tableau 1). Cinq tombes recelaient une épée de fer ( ${ }^{\text {os }} 4-7$ et 12), symbole important de la noblesse kongo illustré sur les gravures d'époque $\left(\mathrm{XVI}^{\mathrm{e}}-\mathrm{XVIII}^{\mathrm{e}}\right.$ siècles), et une sixième renfermait un mousquet $\left(\mathrm{n}^{\circ} 9\right)$. Si l'on se fonde sur ces mêmes gravures, ces attributs seraient caractéristiques de tombes masculines. Des bijoux en or ont été retrouvés dans deux tombes $\left(\mathrm{n}^{\mathrm{os}} 1\right.$ et 8 ) et des médailles religieuses dans trois autres $\left(n^{\text {os }} 2,6\right.$ et 12). Une croix et un crucifix se trouvaient aussi dans la tombe 12. Quatre tombes ont livré des perles en verre $\left(\mathrm{n}^{\mathrm{os}} 8\right.$, 9,11 et 12) qui étaient des symboles de richesse et/ou de féminité [12]. Les tombes 8 et 11 contenaient également 


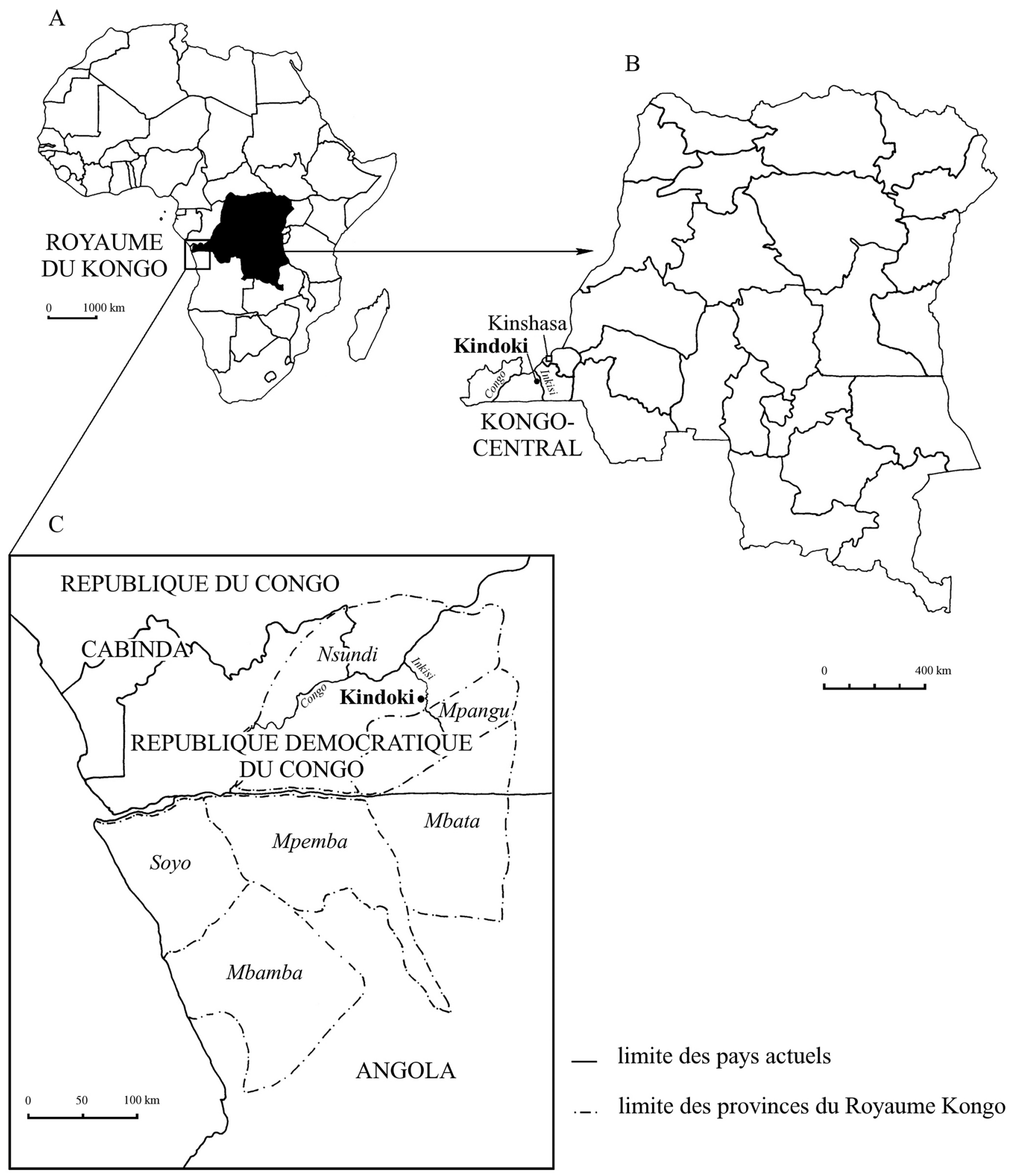

Fig. 1 A. Localisation de la République démocratique du Congo (RDC). B. Carte actuelle de la RDC avec localisation du site de Kindoki dans la province du Kongo central. C. Royaume du Kongo et délimitation de ses provinces aux XVI ${ }^{\mathrm{e}}-\mathrm{XVII}{ }^{\mathrm{e}}$ siècles (dessin : A.-M. Wittek [ADIA], d'après Clist et al. [2]) / A. Location of the Democratic Republic of the Congo (DRC). B. Current map of the DRC with the location of the Kindoki site in the province of Central Kongo. C. Kingdom of Kongo and its boundaries in the 16-17th centuries (drawing : A.-M. Wittek (ADIA) from Clist et al [2]) 


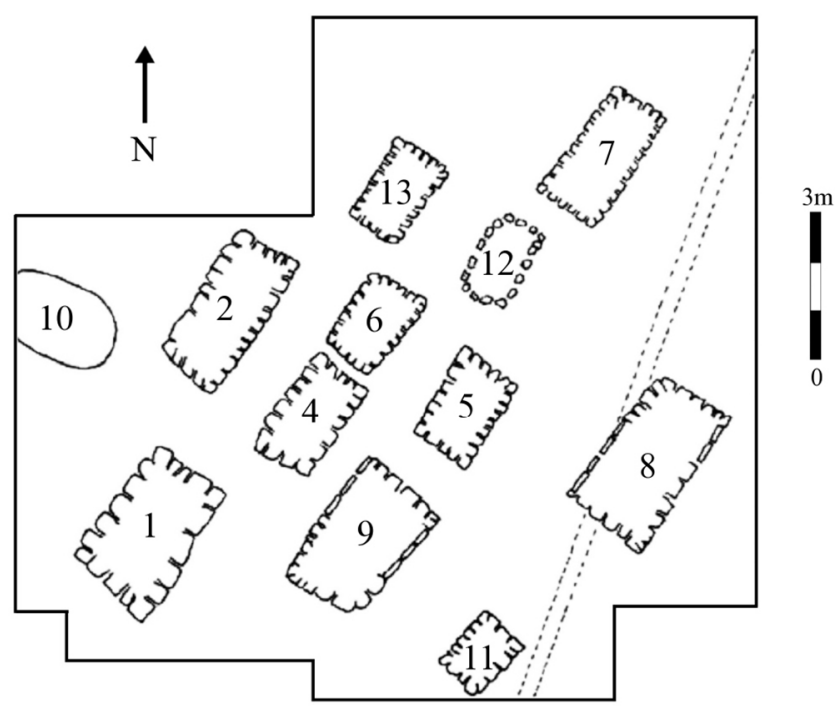

Fig. 2 Plan du cimetière de Kindoki [2] / Map of the cemetery at Kindoki [2] des perles en coquilles de gastéropodes marins ainsi que des chevillères en fer d'un modèle identique. Les armes, les médailles, la croix, le crucifix et les perles de verre sont d'origine européenne. Cela n'est pas surprenant, car le royaume Kongo était en contact avec les Portugais dès 1483, et depuis lors, les échanges de biens avec les Européens étaient largement répandus [13]. La présence de médailles religieuses, de croix et de crucifix n'est, elle aussi, pas inattendue étant donné que les Bakongo se sont graduellement volontairement christianisés à la suite de la conversion du roi Joao $\mathrm{I}^{\text {er }}$ en 1491 [14].

L'étude du mobilier funéraire a permis de proposer des dates dites terminus post quem, à savoir les dates à partir desquelles les inhumations ont pu avoir lieu [2] (Tableau 1). Deux tombes ( ${ }^{\text {os }} 5$ et 9 ) renfermaient du charbon de bois qui a été daté par le radiocarbone (Tableau 1). Les dates calibrées à deux sigma, en tenant compte des tables de calibration de l'hémisphère sud avec le programme Calib 7.0.4., ont donné :

Poz-60769 (tombe 5) : $200 \pm 30$ BP. 1658AD-1712AD (25 \%), 1718AD-1814AD (56 \%), 1835AD-1891AD

Tableau 1 Résultat de l'étude archéologique des 11 tombes de Kindoki : composition du mobilier funéraire, attributions chronologique et sexuelle (genre) / Result of the archaeological study of the 11 graves from Kindoki: composition of the grave goods, chronological and gender attributions

\begin{tabular}{|c|c|c|c|}
\hline Tombe & Mobilier funéraire & Chronologie & Genre \\
\hline 1 & - & - & - \\
\hline 2 & $\begin{array}{l}1 \text { médaille religieuse, } 1 \text { chaîne en or (perdues } \\
\text { sur le chantier) }\end{array}$ & $?$ & $?$ \\
\hline 4 & 1 épée & Fin XVII ${ }^{\mathrm{e}}-$ début XVIII ${ }^{\mathrm{e}}$ siècle (épée) & $\hat{0}$ \\
\hline 5 & 1 épée & $\begin{array}{l}1658-1814\left({ }^{14} \mathrm{C} ; 81 \% \text { de probabilité), XVII }{ }^{\mathrm{e}}-\right. \\
\mathrm{XVIII}^{\mathrm{e}} \text { siècles (épée) }\end{array}$ & 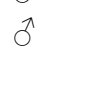 \\
\hline 6 & 1 médaille religieuse, 1 épée & $\begin{array}{l}\text { fin XVIII -début XIX }{ }^{\mathrm{e}} \text { siècle (médaille), XVI }{ }^{\mathrm{e}}- \\
\mathrm{XVII}^{\mathrm{e}} \text { siècles (épée) }\end{array}$ & $\hat{\sigma}$ \\
\hline 7 & 1 épée, 2 fragments de métal en fer & $\mathrm{XVII}^{\mathrm{e}}$ siècle (épée) & $? / \widehat{\jmath}$ \\
\hline 8 & $\begin{array}{l}1159 \text { perles de verre, } 1 \text { perle en cuivre, } 32 \text { cloches } \\
\text { crotales, } 660 \text { perles de coquillage } \\
\text { (Pusula depauperata), } 1 \text { coquillage des mangroves } \\
\text { (Tympanotonus fuscatus radula), } 1 \text { chevillère en fer, } \\
1 \text { collier en fer, } 1 \text { chaîne en cuivre, } 1 \text { chaîne en or, } \\
\text { plusieurs fragments d'un linceul }\end{array}$ & $1825-1845$ (perles) & q \\
\hline 9 & 1 mousquet, 2 bracelets en fer, 18 perles de verre & $\begin{array}{l}1690-1725 \text { (mousquet), } \\
1725-1850 \text { (perles), } 1665-1816\left({ }^{14} \mathrm{C} ; 71 \%\right. \\
\text { de probabilité) }\end{array}$ & 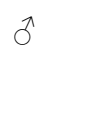 \\
\hline 11 & $\begin{array}{l}268 \text { perles de verre, } 272 \text { perles de coquillage } \\
\text { (Pusula depauperata), } 6 \text { bracelets de cheville en fer }\end{array}$ & $1825-1845$ (perles) & $q$ \\
\hline 12 & $\begin{array}{l}16 \text { perles de verre, } 2 \text { perles d'ivoire, } 1 \text { crucifix } \\
\text { en cuivre, } 1 \text { croix en cuivre, } 1 \text { médaille religieuse, } \\
1 \text { chaîne en cuivre, } 2 \text { fragments de fer, } 1 \text { épée }\end{array}$ & $\begin{array}{l}1650-1750 \text { (perles), XVII }{ }^{\mathrm{e}}-\mathrm{XVIII}^{\mathrm{e}} \text { siècles (crucifix), } \\
\mathrm{XVII}^{\mathrm{e}} \text { siècle (médaille), XVII }{ }^{\mathrm{e}} \text { siècle (épée) }\end{array}$ & $\hat{0}$ \\
\hline 13 & - & - & - \\
\hline
\end{tabular}


(13\%) et 1924AD-1949 AD (6\%). Bêta-333285 (tombe 9) : $190 \pm 30$ BP. 1665AD-1816AD (71\%), 1830AD-1892AD $(20 \%)$ et 1922AD-1949AD (9\%).

La présence dans plusieurs sépultures de vestiges ou d'objets connotant un statut social très élevé dans la tradition kongo actuelle et subactuelle, de nobles dans l'esprit du royaume Kongo (épées, couches multiples de tissus, bracelets de fer au bras droit, centaines de perles en verre et en coquilles marines), amène à conclure que nous avons affaire à des nobles kongo, sinon à des tombes ducales datées entre la fin du XvII ${ }^{\mathrm{e}}$ et le début du $\mathrm{XIX}^{\mathrm{e}}$ siècle [2].

\section{Méthodes}

Pour quantifier l'état de préservation des squelettes, nous avons utilisé l'indice de conservation anatomique (ICA) de Dutour [15] (pp. 26-29). Afin de décrire l'état de préservation des surfaces osseuses, nous avons appliqué la méthode de McKinley [16].

Le sexe n'a malheureusement pas pu être déterminé à partir des os coxaux, car ils sont généralement très fragmentés, voire absents. Nous avons donc utilisé le bloc craniofacial, la mandibule, les os longs et les dents. Les déterminations du sexe à partir de la tête osseuse se sont fondées sur des critères morphologiques et métriques. Pour le bloc craniofacial, nous avons appliqué la méthode de Ferembach et al. [17]. Pour la mandibule, nous avons appliqué la méthode de Gilles citée par Krogman et İșcan [18] (p. 200), mise au point sur des Afro-Américains, et celle de Franklin et al. calculée pour des Zoulous d'Afrique du Sud [19]. Les diagnostics réalisés à partir des os longs ont fait appel à des fonctions discriminantes, comme celles de Dibennardo et Taylor calculées sur la base de fémurs d'Afro-Américains [20] et celles d'İscan et Miller-Shaivitz établies sur des tibias d'Afro-Américains [21]. Les déterminations du sexe fondées sur les dents ont été effectuées grâce :

- aux fonctions discriminantes de Macaluso fondées sur deux mesures réalisées sur les M1 et M2 supérieures de Noirs sud-africains [22];

- à la méthode graphique d'Orban et al., mise au point sur une population européenne et faisant appel à deux mesures relevées sur la PM2 supérieure et la $\mathrm{C}$ inférieure [23].

En fonction de l'état de préservation des individus, les estimations de l'âge au décès ont été fondées sur l'usure dentaire [24], l'oblitération des sutures crâniennes [18] (pp. 120-121), l'ossification du cartilage thyroïdien [18] (pp. 127-129) et l'état de fusion des épiphyses [25] (pp. 293-295).

Les statures ont été estimées à partir de la longueur des os longs, grâce aux formules de Trotter et Gleser destinées aux femmes et aux hommes afro-américains [26]. Lorsque les os étaient incomplets, nous avons appliqué la méthode de Steele et McKern pour estimer leur longueur [27].

Pour élaborer le profil biologique, nous avons privilégié des méthodes anthropologiques mises au point à partir de populations de référence africaines. En l'absence de cellesci, nous avons employé des méthodes largement utilisées en anthropologie biologique. Se pose dans ce cas la question de la transposition à des échantillons africains. Ce point est particulièrement crucial dans le cas de l'usure dentaire, puisqu'elle dépend principalement du régime alimentaire. Comme les chasseurs-cueilleurs amérindiens étudiés par Lovejoy [24] n'avaient pas le même régime que les agriculteurs-éleveurs de Kindoki, les stades d'usure dentaire ne seront pas utilisés pour leur attribuer un âge précis, mais pour les répartir en trois classes d'âge (16-25 ans, 26-40 ans et $>40$ ans), en tenant également compte des résultats obtenus par les autres méthodes d'estimation de l'âge et de la présence de pathologies dégénératives.

Les pathologies osseuses traumatiques, infectieuses, métaboliques et dégénératives ainsi que les pathologies dentaires ont été étudiées à l'échelle macroscopique [28-31]. Les variations anatomiques dentaires et osseuses ont également été examinées [32] tout comme les marqueurs d'activité [33].

Des analyses par microscopie électronique à balayage couplée à la microanalyse par spectroscopie à rayons $\mathrm{X}$ à dispersion d'énergie (MEB-EDS = FEI Quanta 200 équipé de système EDAX, modèle Apollo 10 SDD $^{\circledR}$ Silicon Drift Detectors, $23 \mathrm{kV}$ ) ont été effectuées à l'IRSNB, afin de déterminer l'origine des colorations que présentent certains restes osseux et dentaires [34].

\section{Résultats}

Neuf des 11 sépultures du cimetière de Kindoki ont livré des restes humains (les tombes 4 et 13 en étaient dépourvues). Sept d'entre elles ne renfermaient qu'un seul défunt $(1,2,5$, $6,9,11$ et 12). La tombe 7 contenait des vestiges appartenant à deux individus (7A et 7B).

Une monographie présentant les résultats du projet KongoKing est en préparation [35]. Le chapitre consacré à l'étude des squelettes de Kindoki reprendra l'étude descriptive détaillée de chaque individu, les mesures osseuses et dentaires individuelles ainsi que de nombreuses photographies.

\section{État de préservation}

Les défunts des tombes $1,2,5,6,7$ et 12 sont très incomplets et très fragmentaires. Ils ne sont généralement constitués que de restes dentaires, de fragments de diaphyse et de la tête osseuse (Tableau 2). Leur ICA varie entre 1,1 et $10,2 \%$ (Tableau 2), ce qui correspond à un état de conservation 
Tableau 2 Résultats de l'étude anthropologique des dix individus de Kindoki : inventaire des restes dentaires et osseux $(\mathrm{F}=$ os incomplet, $\mathrm{FF}=$ quelques fragments osseux), indice de conservation anatomique (ICA), état de préservation des surfaces osseuses (PSO), détermination du sexe, stade d'usure dentaire de Lovejoy [24], classe d'âge, pathologie et particularité anatomique / Results of the anthropological study of the 10 individuals from Kindoki: dental and bone inventory $(\mathrm{F}=$ incomplete bone, $\mathrm{FF}=$ several bone fragments), Anatomical Preservation Index (ICA), bone surface preservation (PSO), sex determination, Lovejoy dental wear phase [24], age class, age estimation, pathology and anatomically distinctive traits

\begin{tabular}{|c|c|c|c|c|c|c|c|c|c|c|c|}
\hline $\begin{array}{l}\text { Individu } \\
n^{0}\end{array}$ & $\begin{array}{l}N \\
\text { dents }\end{array}$ & $\begin{array}{l}\text { Os } \\
\text { long }\end{array}$ & $\begin{array}{l}\text { Tête } \\
\text { osseuse }\end{array}$ & $\begin{array}{l}\text { Autres } \\
\text { os }\end{array}$ & $\begin{array}{l}\text { ICA } \\
(\%)\end{array}$ & PSO & Sexe & $\begin{array}{l}\text { Usure } \\
\text { dentaire }\end{array}$ & $\begin{array}{l}\text { Classe } \\
\text { d'âge } \\
\text { (années) }\end{array}$ & Pathologie & Particularité \\
\hline 1 & 30 & FF & $\mathrm{FF}$ & - & 10,2 & 5 & 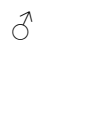 & $\mathrm{F}$ & $26-40$ & Carie, tartre & $\begin{array}{l}\text { Torus } \\
\text { mandibulaire, } \\
\text { usure pipe }\end{array}$ \\
\hline 2 & 15 & $\mathrm{FF}$ & $\mathrm{FF}$ & - & 4,5 & $5+$ & $\sigma^{\lambda}$ & $\mathrm{G}-\mathrm{H}$ & $26-40$ & - & - \\
\hline 5 & 22 & $\mathrm{FF}$ & - & - & 4,0 & $5+$ & $\widehat{\sigma}$ & $\mathrm{E}$ & $26-40$ & Tartre & Usure pipe \\
\hline 6 & 11 & - & - & - & 2,3 & - & $?$ & $\mathrm{C}$ & $16-25$ & - & - \\
\hline $7 \mathrm{~A}$ & 12 & FF & FF & - & 2,8 & $5+$ & $\widehat{\sigma}$ & $\mathrm{G}$ & $26-40$ & - & LSAMAT \\
\hline $7 \mathrm{~B}$ & 12 & - & - & - & 1,1 & - & 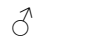 & $>\mathrm{I}$ & $>40$ & - & - \\
\hline 8 & 31 & $\mathrm{~F}$ & $\mathrm{~F}$ & $\mathrm{~F}$ & 34,1 & $3-5$ & 우 & $\mathrm{H}$ & $>40$ & $\begin{array}{l}\text { Parodontite, } \\
\text { DISH ? } \\
\text { Arthrose, } \\
\text { tartre }\end{array}$ & $\begin{array}{l}13 \text { vertèbres } \\
\text { thoraciques, } \\
\text { LSAMAT }\end{array}$ \\
\hline 9 & 24 & $\mathrm{~F}$ & $\mathrm{~F}$ & $\mathrm{~F}$ & 43,2 & $2-3$ & $\sigma^{\lambda}$ & G & $>40$ & $\begin{array}{l}\text { Carie, } \\
\text { arthrose }\end{array}$ & $\begin{array}{l}\text { Suture } \\
\text { métopique }\end{array}$ \\
\hline 11 & 25 & $\mathrm{~F}$ & $\mathrm{~F}$ & $\mathrm{~F}$ & 38,1 & $1-3$ & 우 & $\mathrm{H}$ & $>40$ & $\begin{array}{l}\text { Parodontite, } \\
\text { DISH, } \\
\text { arthrose }\end{array}$ & $\begin{array}{l}\text { Courbure } \\
\text { du fémur }\end{array}$ \\
\hline 12 & 6 & $\mathrm{FF}$ & $\mathrm{FF}$ & FF & 4 & $1-3$ & $?$ & $\mathrm{~B} 2-\mathrm{C}$ & $16-25$ & - & - \\
\hline
\end{tabular}

allant de mauvais à médiocre [15]. De plus, leurs surfaces osseuses sont très érodées (Fig. 3, Tableau 2). On note la présence de fissures dans presque toutes les couronnes dentaires. Les racines sont friables, souvent très érodées et parfois même absentes (individus 5, 6 et 7B).

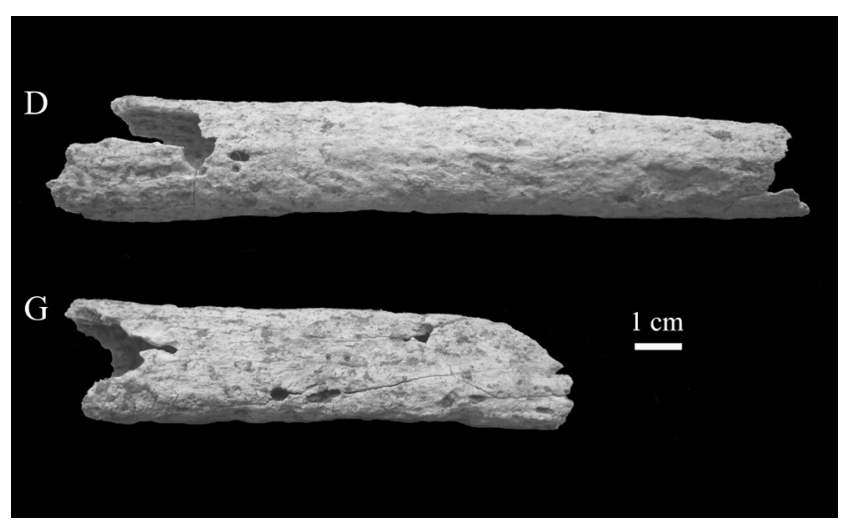

Fig. 3 Tibias droit (D) et gauche (G) de l'individu de la tombe 2 de Kindoki. Ils se caractérisent par une très forte érosion des surfaces / Right (D) and left (G) tibias of individual 2 from Kindoki. Both have severely eroded surfaces
Les squelettes des tombes 8,9 et 11 situées à l'est du cimetière sont bien mieux conservés que les précédents (Tableau 2). Leurs ICA varient entre 34,1 et $43,21 \%$, ce qui correspond à un assez bon à bon état de conservation [15]. Ils sont néanmoins incomplets, de nombreux ossements sont fragmentaires (Annexes A-C), et leurs surfaces sont érodées (Tableau 2). Les os des mains et des pieds (sauf pour l'individu 9), les patellas et le sacrum font défaut. Les épiphyses des os longs sont aussi généralement absentes. Les couronnes dentaires sont fissurées et ont perdu de nombreux éclats d'émail. Les racines sont légèrement érodées.

Chez l'individu 9, une concrétion métallique (oxyde de fer) en forme d'anneau entoure la diaphyse humérale droite au niveau de son tiers supérieur (Annexe B, Fig. 4).

\section{Coloration des restes humains}

Les squelettes 8,9 et 11 présentent des colorations vertes (Annexes A-C). Chez l'individu 9, elles concernent même les dents. Les analyses au MEB-EDS montrent que ces colorations sont dues à la présence d'un ou de plusieurs oxydes de cuivre. 


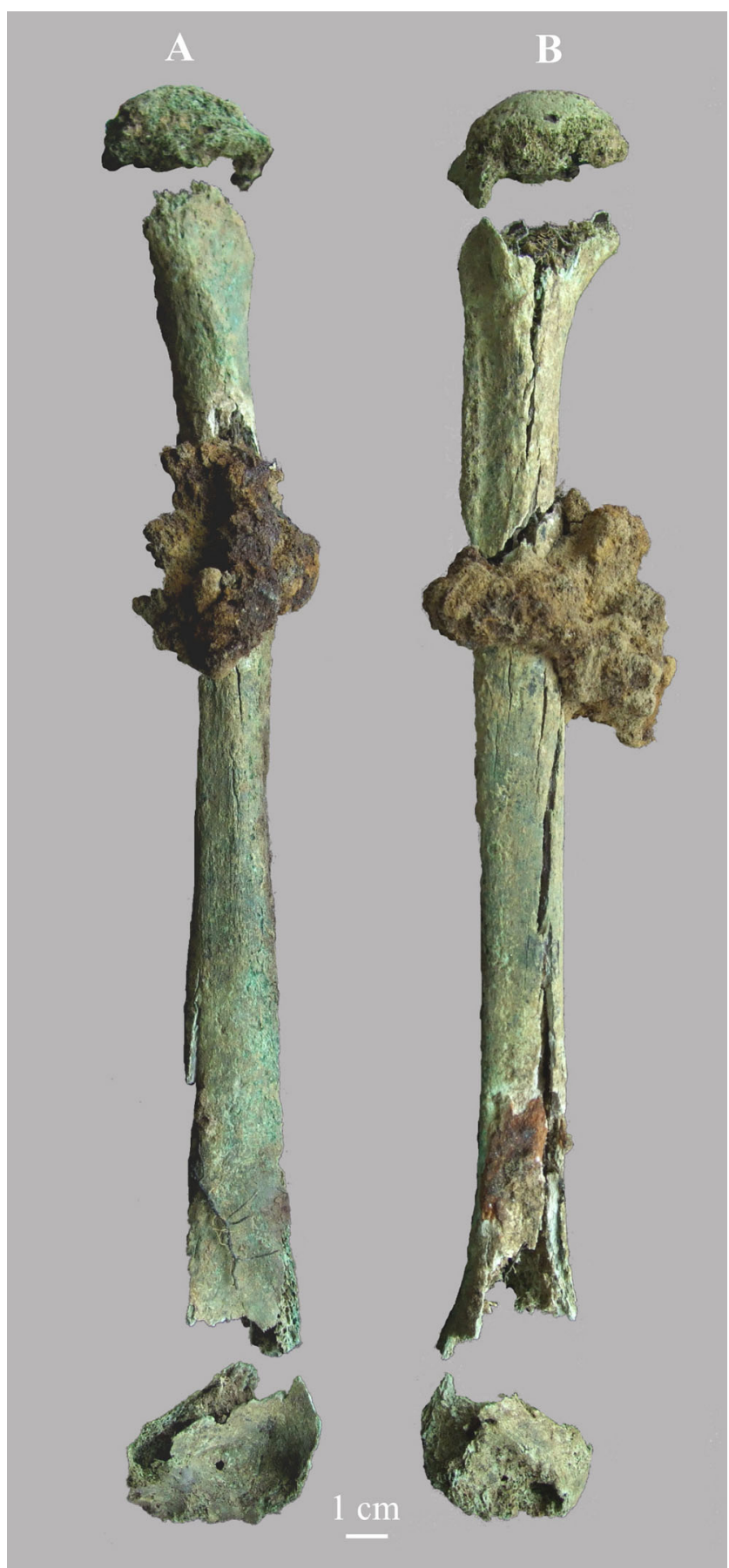

Fig. 4 Humérus droit de l'individu de la tombe 9 de Kindoki. A. Vue antérieure. B. Vue postérieure / Right humerus from the individual in grave $\mathrm{n}^{\circ} 9$ at Kindoki. A. Anterior view. B. Posterior view

L'individu 8 se caractérise également par des colorations roses (Annexe A). Les analyses au MEB-EDS ont révélé des teneurs élevées en plomb et en fer. Le plomb n'est pas responsable de cette coloration. Des oxydes de fer à faible concentration pourraient être à l'origine des teintes roses.

Les squelettes 9 et 11 présentent aussi des teintes brun foncé au niveau des membres inférieurs (Annexes B,C).
Les analyses au MEB-EDS montrent que ces colorations sont liées à la présence d'oxydes de fer.

\section{Détermination du sexe}

Les déterminations du sexe des individus $1,2,5,6,7$ et 12 ont été réalisées uniquement à partir des mensurations dentaires. Celles des individus 8,9 et 11 ont aussi fait appel à des méthodes fondées sur la tête osseuse et les os longs (Tableau 3).

Six individus ont été diagnostiqués comme masculins : 1 , 2, 5, 7A, 7B et 9. Les défunts des tombes 8 et 11 ont été déterminés comme étant de sexe féminin. Les individus 6 et 12 sont de sexe indéterminé (Tableau 2).

\section{Estimation de l'âge au décès}

La présence exclusive de dents permanentes usées (y compris des M3) indique que les individus sont adultes. Seul l'individu 12, qui présente des M1 et M2 peu usées, pourrait être un adolescent.

Pour estimer leur âge au décès, nous nous sommes principalement fondés sur l'usure dentaire (Tableau 4), avec les réserves énoncées dans le chapitre " Méthodes 》. Pour les individus des tombes 8,11 et 12 , nous avons aussi employé respectivement l'ossification du cartilage du larynx, l'oblitération des sutures crâniennes et l'état de fusion de l'épiphyse distale du radius (Tableau 4). Les résultats sont rassemblés dans le tableau 2.

Les individus 5, 6 et 12 seraient les plus jeunes, alors que les individus 7B, 8 et 11 seraient les plus âgés.

Le fait que les individus 8 et 9 présentent des atteintes arthrosiques de la colonne vertébrale (voir ci-dessous) indique, par analogie aux connaissances cliniques actuelles, qu'il s'agirait d'adultes ayant au moins atteint 40 ans [3638] (p. 459 de [37]).

\section{Estimation de la stature}

Les statures n'ont pu être estimées que pour les trois individus les mieux préservés.

La stature de l'individu 11 calculée à partir de la longueur $\mathrm{du}$ fémur vaut $155,3 \pm 3,41 \mathrm{~cm}$.

L'estimation de la stature des individus 8 et 9 a nécessité deux étapes : l'estimation de la longueur du fémur et l'estimation de la stature. L'erreur associée à l'estimation de la longueur du fémur est de $1,31 \mathrm{~cm}$ pour les sujets masculins et de 1,02 cm pour les sujets féminins [27]. La stature de la femme de la tombe 8 vaut $157,2 \pm 3,41 \mathrm{~cm}$ (cette valeur est probablement sous-estimée étant donné que l'individu présentait une vertèbre surnuméraire). L'homme de la tombe 9 aurait mesuré $161,5 \pm 3,94 \mathrm{~cm}$. 
Tableau 3 Méthodes appliquées pour déterminer le sexe des dix individus de Kindoki / Methods used to determine the sex of the 10 individuals from Kindoki

\begin{tabular}{|c|c|c|c|c|c|c|c|}
\hline \multirow[b]{2}{*}{ Individu $\mathbf{n}^{\circ}$} & \multicolumn{2}{|c|}{ Dents (biométrie) } & \multirow{2}{*}{$\begin{array}{l}\text { Bloc } \\
\text { craniofacial } \\
\text { Morphologie } \\
\text { [17] }\end{array}$} & \multicolumn{2}{|c|}{ Mandibule } & \multicolumn{2}{|c|}{ Os longs (biométrie) } \\
\hline & Molaires [22] & $\begin{array}{l}\text { Prémolaire } \\
\text { et canine [23] }\end{array}$ & & $\begin{array}{l}\text { Biométrie [18] } \\
\text { (p. 200) }\end{array}$ & Biométrie [19] & Fémur [20] & Tibia [21] \\
\hline 1 & $\mathrm{X}$ & $\mathrm{X}$ & & & & & \\
\hline 2 & $\mathrm{X}$ & & & & & & \\
\hline 5 & $\mathrm{X}$ & $\mathrm{X}$ & & & & & \\
\hline 6 & $\mathrm{X}$ & & & & & & \\
\hline $7 \mathrm{~A}$ & $\mathrm{X}$ & & & & & & \\
\hline 7B & $\mathrm{X}$ & & & & & & \\
\hline 8 & $\mathrm{X}$ & $\mathrm{X}$ & $\mathrm{X}$ & $\mathrm{X}$ & $\mathrm{X}$ & $\mathrm{X}$ & $\mathrm{X}$ \\
\hline 9 & $\mathrm{X}$ & & $\mathrm{X}$ & & $\mathrm{X}$ & $\mathrm{X}$ & \\
\hline 11 & $\mathrm{X}$ & $\mathrm{X}$ & $\mathrm{X}$ & $\mathrm{X}$ & & $\mathrm{X}$ & \\
\hline 12 & $\mathrm{X}$ & & & & & & \\
\hline
\end{tabular}

Tableau 4 Méthodes appliquées pour estimer l'âge au décès des dix individus de Kindoki / Methods used to estimate the age at death of the 10 individuals from Kindoki

\begin{tabular}{|c|c|c|c|c|}
\hline Individu $\mathrm{n}^{\mathrm{o}}$ & Usure dentaire [24] & $\begin{array}{l}\text { Sutures crâniennes [18] } \\
\text { (pp. 120-121) }\end{array}$ & $\begin{array}{l}\text { Cartilage thyroïdien } \\
\text { [18] (pp. 127-129) }\end{array}$ & $\begin{array}{l}\text { Fusion épiphyse }[25] \\
\text { (pp. 293-295) }\end{array}$ \\
\hline 1 & $\mathrm{X}$ & & & \\
\hline 2 & $\mathrm{x}$ & & & \\
\hline 5 & $\mathrm{X}$ & & & \\
\hline 6 & $\mathrm{X}$ & & & \\
\hline $7 \mathrm{~A}$ & $\mathrm{x}$ & & & \\
\hline $7 \mathrm{~B}$ & $\mathrm{X}$ & & & \\
\hline 8 & $\mathrm{X}$ & & $\mathrm{X}$ & \\
\hline 9 & $\mathrm{X}$ & & & \\
\hline 11 & $\mathrm{X}$ & $\mathrm{X}$ & & \\
\hline 12 & $\mathrm{x}$ & & & $X$ \\
\hline
\end{tabular}

\section{Pathologie buccodentaire}

Seuls deux individus étaient atteints de caries dentaires. Chez le sujet 1, elle concerne la PM1 inférieure gauche. La lésion a détruit toute la couronne (stade 4 de Metress et Conway [39]) (Fig. 5A). Chez le sujet 9, deux caries sont à signaler : la couronne de la M3 supérieure gauche est à moitié détruite (stade 2), alors que celle de la M2 supérieure gauche a totalement disparu (stade 4). Le taux de dents cariées s'élève à $1,6 \%$ (3/188).

Trois individus présentent des dépôts de tartre dentaire. Chez l'individu 1, le dépôt est léger [31] (pp. 153-154) et concerne la face vestibulaire des PM1 supérieures et la face linguale des dents inférieures (Fig. 5A). Chez l'individu 5, le dépôt est moyen et se situe sur les faces linguales et vestibulaires de la majorité des 22 dents. Chez l'individu 8 , on note un léger dépôt de tartre sur la face linguale des incisives supérieures.

On observe chez les individus 8 et 9 une parodontopathie généralisée. Elle s'exprime par une résorption de l'os alvéolaire [29,30,40] (pp. 260-269 de [30]), qui atteint au moins le stade 1 de Brabant et Sahly (régression sur moins d'un tiers de la racine [41]). Chez l'individu 8, l'os montre une porosité, signe que le processus de remodelage était encore en cours.

On relève également chez l'individu 8 une hypercémentose, c'est-à-dire un épaississement anormal du cément dentaire [30,42] (pp. 205-206 de [30] ; pp. 255-256 de [42]). Elle est particulièrement visible au niveau des canines (Fig. 6B).

Les femmes 8 et 11 présentent des atteintes périapicales. Chez l'individu 8 , on observe six cavités à paroi rugueuse (abcès ? [43]) : au niveau de la M2 et de la $\mathrm{C}$ inférieures 

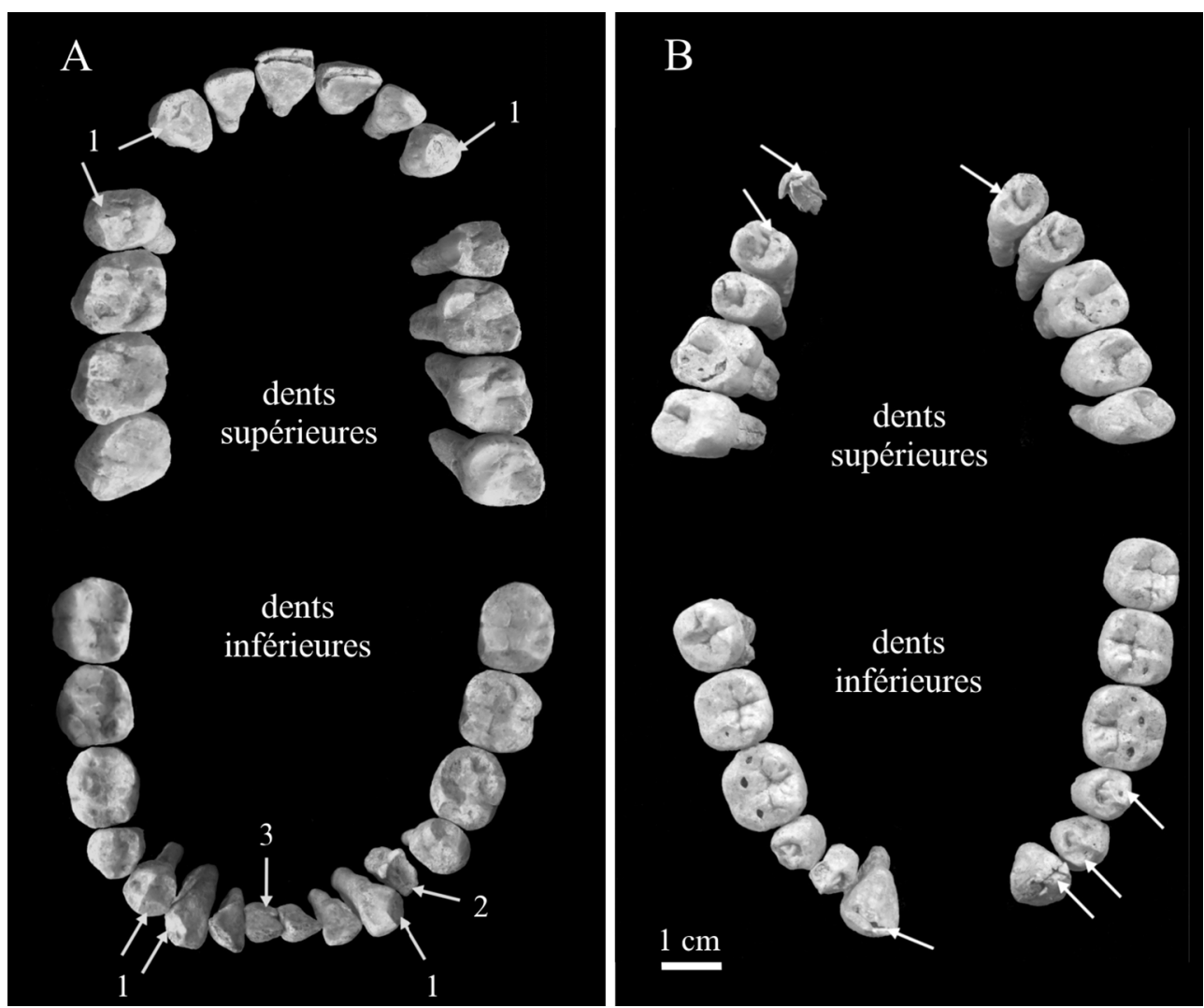

Fig. 5 A. Denture de l'individu de la tombe 1 de Kindoki $(1=$ usures semi-circulaires ; 2 = carie ; $3=$ tartre $)$. B. Denture de l'individu de la tombe 5 de Kindoki. Les flèches indiquent la localisation des usures semi-circulaires / A. Teeth of the individual from grave $\mathrm{n}^{\mathrm{o}} 1$ at Kindoki $(1=$ semicircular wear, $2=$ caries, $3=$ dental calculus $)$. B. Teeth of the individual from grave $\mathrm{n}^{\mathrm{o}} 5$ at Kindoki. The arrows show the location of semicircular wear

droites (Fig. 6A), de la M1 (avec perforation du plancher du sinus) et de la $\mathrm{C}$ supérieures droites, des M1 et M3 supérieures gauches (Fig. 6B). L'individu 11 présente deux cavités périapicales à paroi lisse (kyste ? [43]) (Fig. 7) : au niveau de la PM1 et de la M2 inférieures gauches. Dans ce dernier cas, la destruction de l'os alvéolaire est telle qu'elle a permis le déplacement de cette molaire. Elle a pu pencher légèrement mésialement (Fig. 7Ba) ou totalement basculer jusqu'à reposer sa face mésiale sur l'os alvéolaire (Fig. 7Bb). Elle n'était, de toute façon, plus en contact avec la dent antagoniste supérieure, étant donné qu'elle est nettement moins usée que sa correspondante droite. Les bascules antérieures sont habituellement observées lors de la perte d'une molaire : la molaire suivante bascule vers le site de la dent manquante [44]. Ce n'est pas le cas de notre sujet, puisque sa M1 est présente et en place : c'est l'espace dégagé postérieurement par la destruction de l'alvéole (Fig. 7Bc) qui a rendu possible le déplacement de la dent.

Chez l'individu 11, on note la présence d'une ligne d'hypoplasie de l'émail dentaire [30] (pp. 165-177) sur les PM1 et PM2 inférieures gauches (la droite est trop mal préservée), les M1 et M2 inférieures droites, la M2 supérieure gauche et les M3 (Fig. 7A).

\section{Usures dentaires particulières}

Deux individus de sexe masculin présentent des usures dentaires de forme semi-circulaire. Chez l'individu 1, elles concernent les C, la PM1 inférieure droite (la gauche a été détruite par une carie, et les PM1 supérieures sont manquantes) et la PM2 supérieure droite (Fig. 5A). Chez l'individu 5, les usures touchent les canines, les prémolaires et les premières molaires (Fig. 5B).

Les incisives supérieures des individus $7 \mathrm{~A}$ et 8 présentent une usure qui s'étend sur leur face linguale. Ce type d'usure des dents antérosupérieures a été dénommé LSAMAT (lingual surface attrition of the maxillary anterior teeth) [45].

\section{Pathologie osseuse}

Les deuxième et troisième vertèbres thoraciques de l'individu 8 présentent une zone de fusion (Fig. 8A). Cette fusion est localisée antérieurement et restreinte au côté droit des corps vertébraux. L'espace entre les deux vertèbres est conservé. Deux côtes droites sont fusionnées avec les corps vertébraux sur lesquels elles s'articulent (Fig. 8B). 

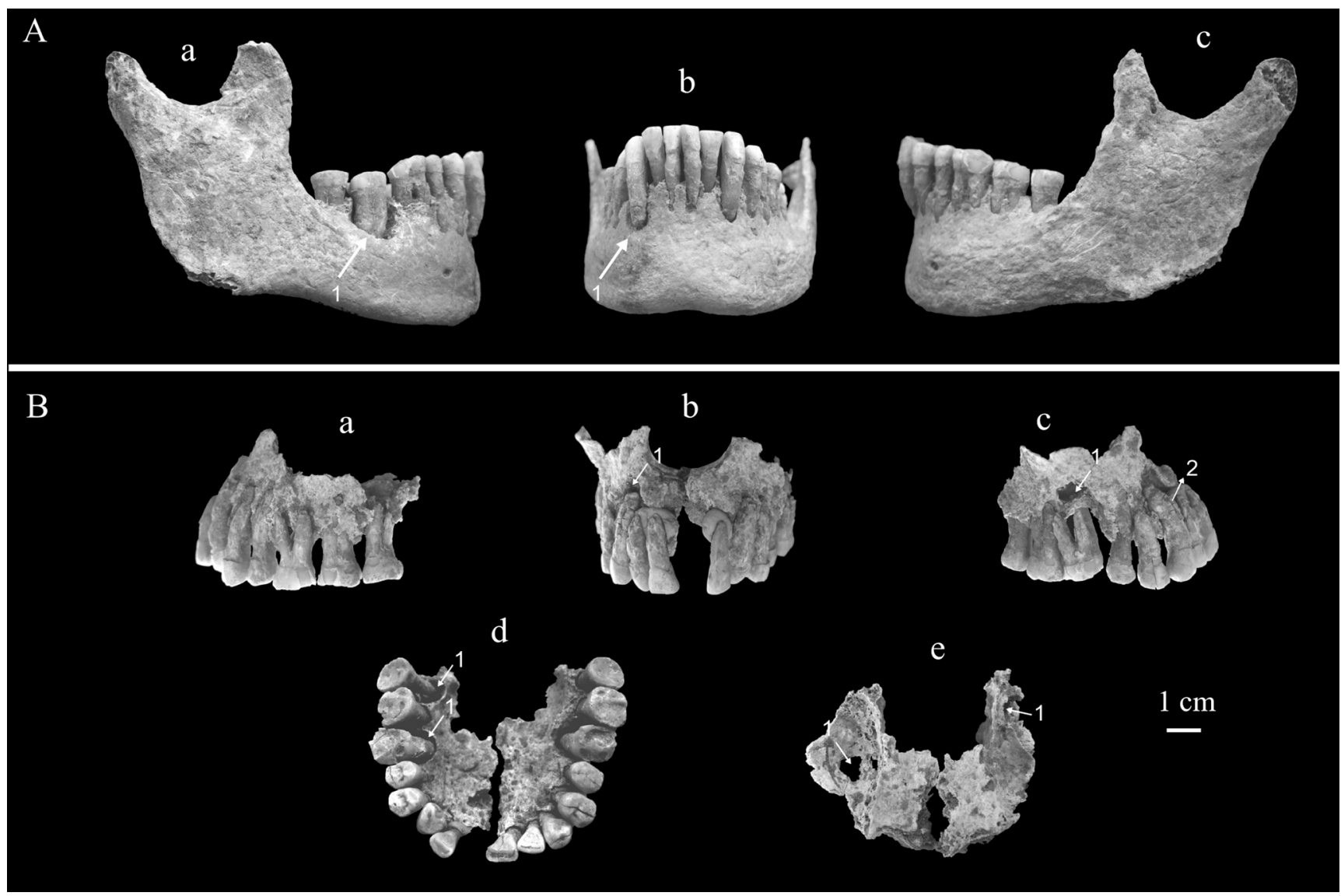

Fig. 6 Mandibule et maxillaires de l'individu de la tombe 8 de Kindoki. Les flèches indiquent la localisation des atteintes périapicales (1) et l'hypercémentose (2). A. Mandibule ; a : vue latérale droite ; b : vue antérieure ; $:$ vue latérale gauche. B. Maxillaires (de la plasticine a été appliquée pour fixer les dents antérieures) ; a : vue latérale gauche ; b : vue antérieure ; $\mathrm{c}$ : vue latérale droite ; d : vue inférieure ; e : vue supérieure / Mandible and maxillae of the individual from grave $n^{\circ} 8$ at Kindoki. The arrows show the location of the periapical lesions (1) and hypercementosis (2). A. Mandible; a: right side view; b: anterior view; c: left side view. B. Maxillae (plasticine was applied to fix the anterior teeth); a: left side view; b: anterior view; c: right side view; d: inferior view; e: superior view

Au moins sept vertèbres thoraciques de la femme de la tombe 11 montrent des zones de fusion (Fig. 9). Comme dans le cas de l'individu 8, les fusions sont localisées antérieurement et restreintes au côté droit des corps vertébraux. Elles s'expriment sous forme de ponts osseux qui ont l'aspect d'une coulée de cire. Les espaces entre les vertèbres sont conservés.

Les vertèbres de l'individu 8 présentent des signes d'ostéoarthrose modérée qui affecte les différents étages du rachis. Le sujet 9 est aussi atteint de maladie articulaire dégénérative au niveau du seul corps préservé de vertèbre cervicale et au niveau de la cavité glénoïde droite.

\section{Particularités anatomiques}

L'individu 1 présente un torus mandibulaire composé de trois nodules : un situé à droite au niveau de la PM2, deux à gauche, respectivement au niveau de la PM1 et de la PM2.
L'individu 8 se caractérise par 13 vertèbres thoraciques au lieu de 12 (Annexe A). L'os frontal de l'individu 9 comporte une suture métopique. Les fémurs de l'individu 11 accusent une courbure antérieure prononcée.

\section{Discussion}

Neuf des 11 tombes de la colline de Kindoki ont livré des restes humains, mais la majorité présente un très mauvais état de conservation. Ces détériorations sont à imputer aux conditions d'enfouissement des sites de plein air localisés dans cette région au climat tropical humide (province du Kongo central et province du Zaïre en Angola) [46]. Le degré de préservation serait, de plus, lié à leur durée de séjour dans ce type de sol. Jusqu'à présent, aucun reste humain antérieur au XVII siècle n'y a été retrouvé. Pour ceux datant plus ou moins de 150 ans (comme la tombe 9), l'ICA 

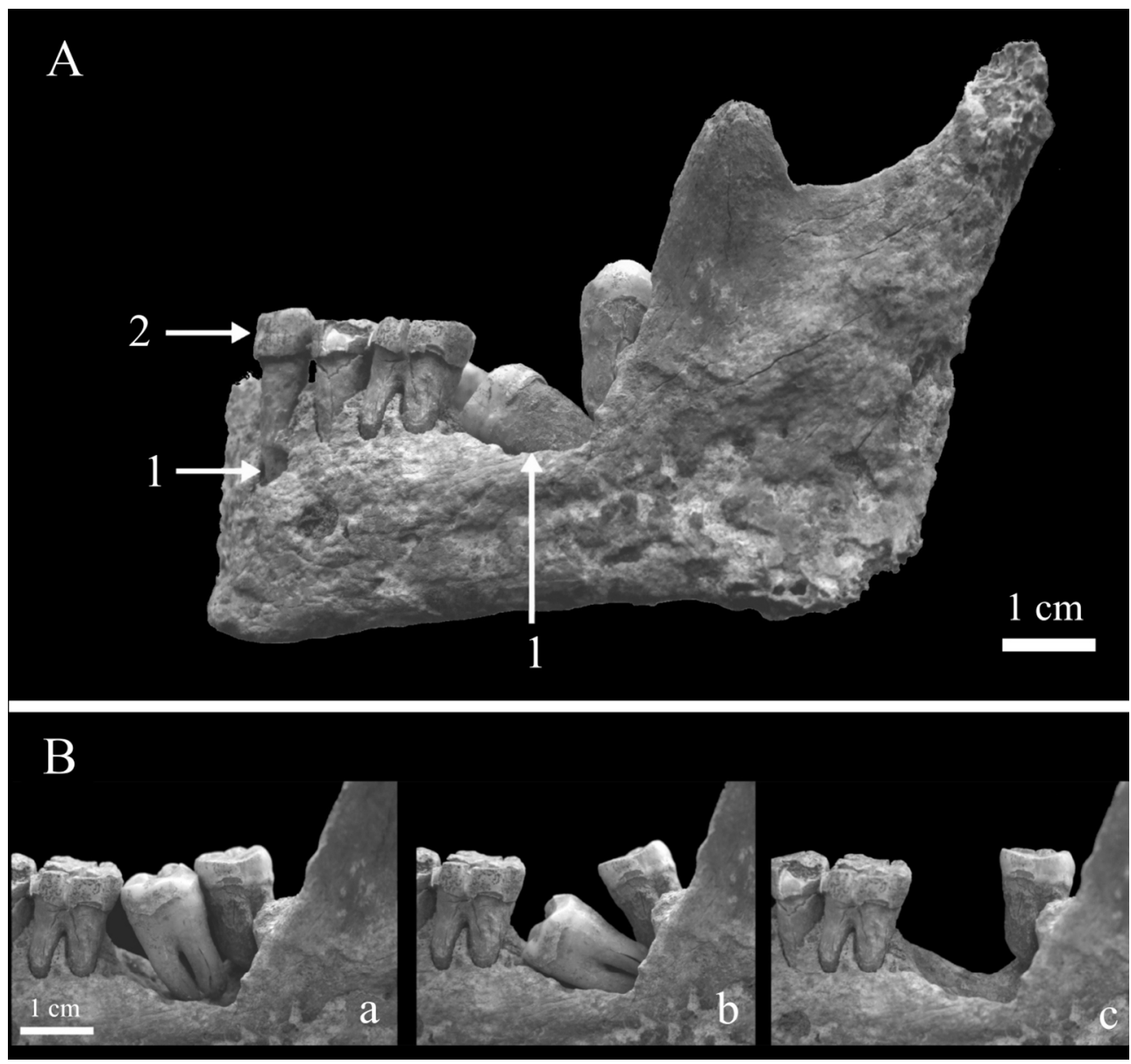

Fig. 7 Mandibule de l'individu de la tombe 11 de Kindoki. A. Vue latérale gauche $(1=$ lésions périapicales, $2=$ ligne d'hypoplasie de l'émail). B. Vues latérales gauches rapprochées. Deux scénarii (a et b) sont proposés en ce qui concerne la position de la seconde molaire ; a : la dent était encore plus ou moins dans sa position initiale ; b : la dent avait totalement basculé sur sa face mésiale ; $\mathrm{c}$ : vue de la cavité sans la deuxième molaire / Mandible of the individual from grave $\mathrm{n}^{\mathrm{o}} 11$ at Kindoki. A. Left side view $(1=$ periapical lesions, 2 = enamel hypoplasia line). B. Close-up lateral views. Two hypotheses ( $\mathrm{a}$ and $\mathrm{b}$ ) are proposed concerning the position of the second molar; a: the tooth was still more or less in its original position ; b: the tooth had twisted entirely onto its mesial face; c: view of the cavity without the second molar

atteint à peine les $40 \%$, et les surfaces osseuses sont généralement dégradées.

L'étude anthropologique est de ce fait très limitée, et les résultats obtenus sont à considérer avec prudence.

Le nombre minimum d'individus s'élève à dix, car la tombe 7 contenait les restes de deux individus. L'étude anthropologique montre que, parmi ces dix individus, six seraient de sexe masculin, deux de sexe féminin et deux de sexe indéterminé. Une comparaison de ces déterminations du sexe avec celles réalisées à partir du mobilier funéraire aboutit à une bonne concordance (Tableaux 1,2). Trois tombes, déterminées comme masculines sur la base de la présence d'armes $(5,7$ et 9), ont livré des restes humains attribués au sexe masculin par l'étude anthropologique. Il en est de même pour les deux tombes diagnostiquées féminines sur la base de l'absence d'arme et la présence de nombreuses perles de verre ( 8 et 11). Les individus 6 et 12, dont l'analyse métrique des restes dentaires n'a pas permis de déterminer le sexe, sont diagnostiqués de sexe masculin sur la base de l'étude du mobilier funéraire. En combinant l'étude des squelettes et du mobilier funéraire, il y aurait donc deux femmes et huit hommes. Les individus les plus jeunes ont été inhumés au centre du cimetière : il s'agit d'adolescents ou de jeunes adultes.

La couleur verte des os des individus 8,9 et 11 et des dents de l'individu 9 peut s'expliquer parfois par la présence d'objets en cuivre dans la tombe (Tableau 1, tombe 8). De même, la couleur brune des os des membres inférieurs des sujets 9 et 11 résulterait de la migration d'oxydes provenant d'objets en fer au contact des défunts (Tableau 1). La teinte rose présente chez l'individu 8 pourrait être aussi liée à la présence d'oxydes de fer ou provenir de colorants présents 

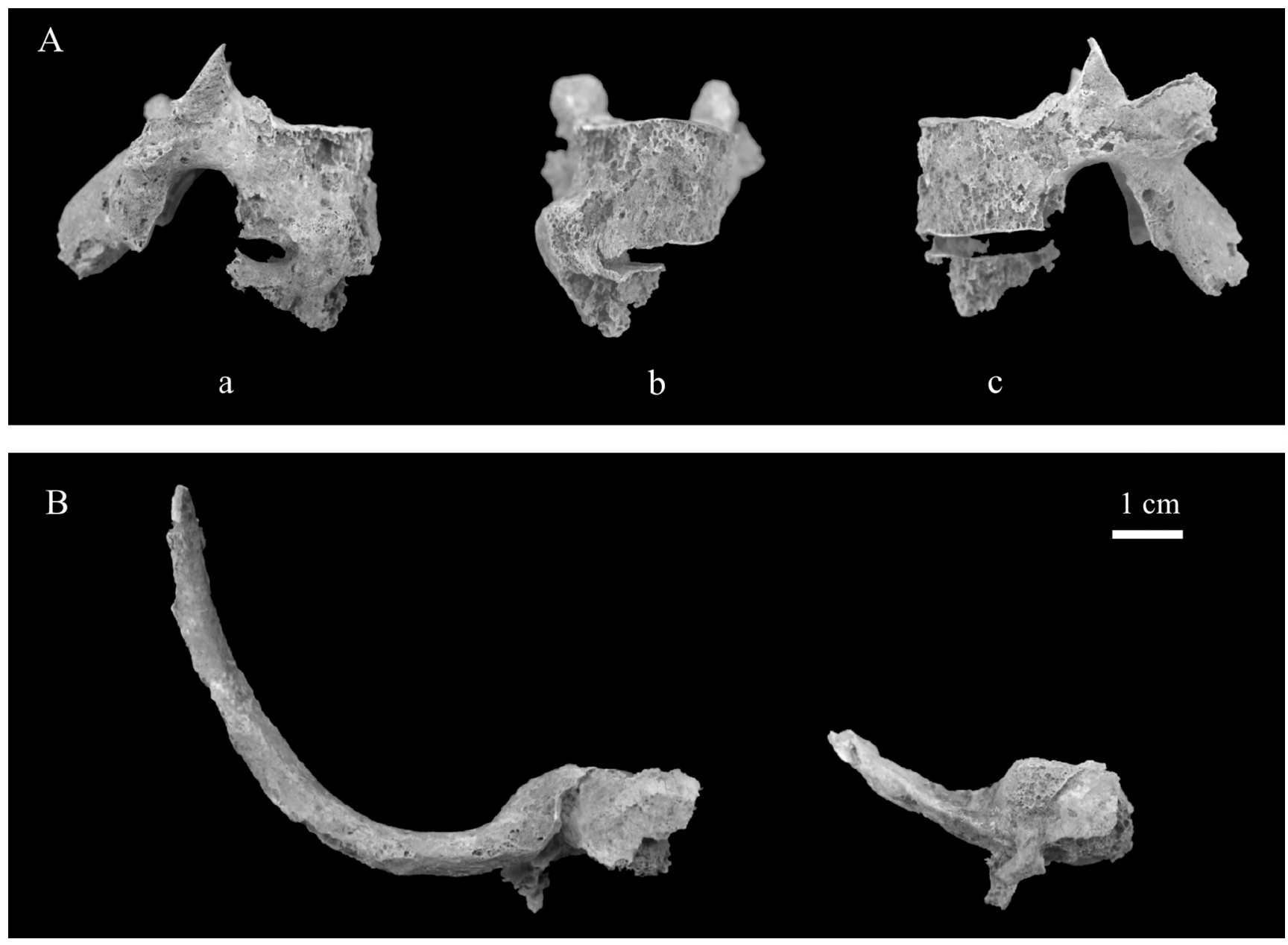

Fig. 8 Vertèbres de l'individu de la tombe $n^{\circ} 8$ de Kindoki. A. Deuxième et troisième vertèbres thoraciques ; $a:$ vue latérale droite ; $b$ : vue antérieure ; $\mathrm{c}$ : vue latérale gauche. B. Vue supérieure des côtes soudées aux dernières vertèbres thoraciques / Vertebrae of the individual from grave 8 at Kindoki. A. Second and third thoracic vertebrae; a: right side view ; b: anterior view ; c: left side view. B. Superior view of the ribs fused to the last thoracic vertebrae

dans les tissus qui entouraient le cadavre (chapitre « Matériel » et Tableau 1).

Quatre particularités osseuses ont été relevées à Kindoki : un torus mandibulaire, une suture métopique, une vertèbre surnuméraire et une courbure accentuée du fémur. Elles seraient d'origine génétique, environnementale [47,48] (pp. 41-44 de [48]) ou, pour la dernière, biomécanique [49]. Reginato et al. [50] mentionnent que le torus affecte 3,2\% des Africains, alors que Corruccini [51] cite une fréquence deux fois plus élevée. La suture métopique est présente chez 1 à $3,4 \%$ des individus africains [52]. La vertèbre surnuméraire serait plus fréquente chez les Africains [32] (pp. 218219). En général, cette variabilité s'accompagne d'un phénomène de compensation : quand il y a une vertèbre thoracique en plus, il y a une vertèbre lombaire en moins [53] (pp. 234235), mais ce n'est pas le cas chez notre individu. Walensky [54] a relevé des valeurs de courbure du fémur les moins élevées chez les individus d'origine africaine. Des caractères discrets ont permis d'identifier des groupements familiaux dans un cimetière soudanais datant $\mathrm{du} \mathrm{III}^{\mathrm{e}}$ au vil $\mathrm{e}^{\mathrm{e}}$ siècle [55]. Ce type d'étude n'est malheureusement pas envisageable à Kindoki, étant donné le faible effectif et la mauvaise préservation des restes osseux.

Les individus masculins des tombes 1 et 5 présentent des usures dentaires semi-circulaires révélatrices de l'utilisation d'une pipe [56]. Cette dernière, surtout lorsqu'elle est en matériau dur, peut en effet entraîner des usures dentaires au niveau des positions préférentiellement adoptées. Fumer le tabac au royaume Kongo est attesté dès 1612 [57], et des exemplaires de pipes en terre cuite et en pierre ont été découverts par l'équipe du projet KongoKing lors des fouilles de la colline de Kindoki [2].

L'étude de la stature ainsi que des pathologies osseuses et dentaires constitue le point de départ d'une recherche plus vaste qui portera sur le régime alimentaire, l'état sanitaire, le statut social et leurs interconnexions. En effet, pour l'instant, 

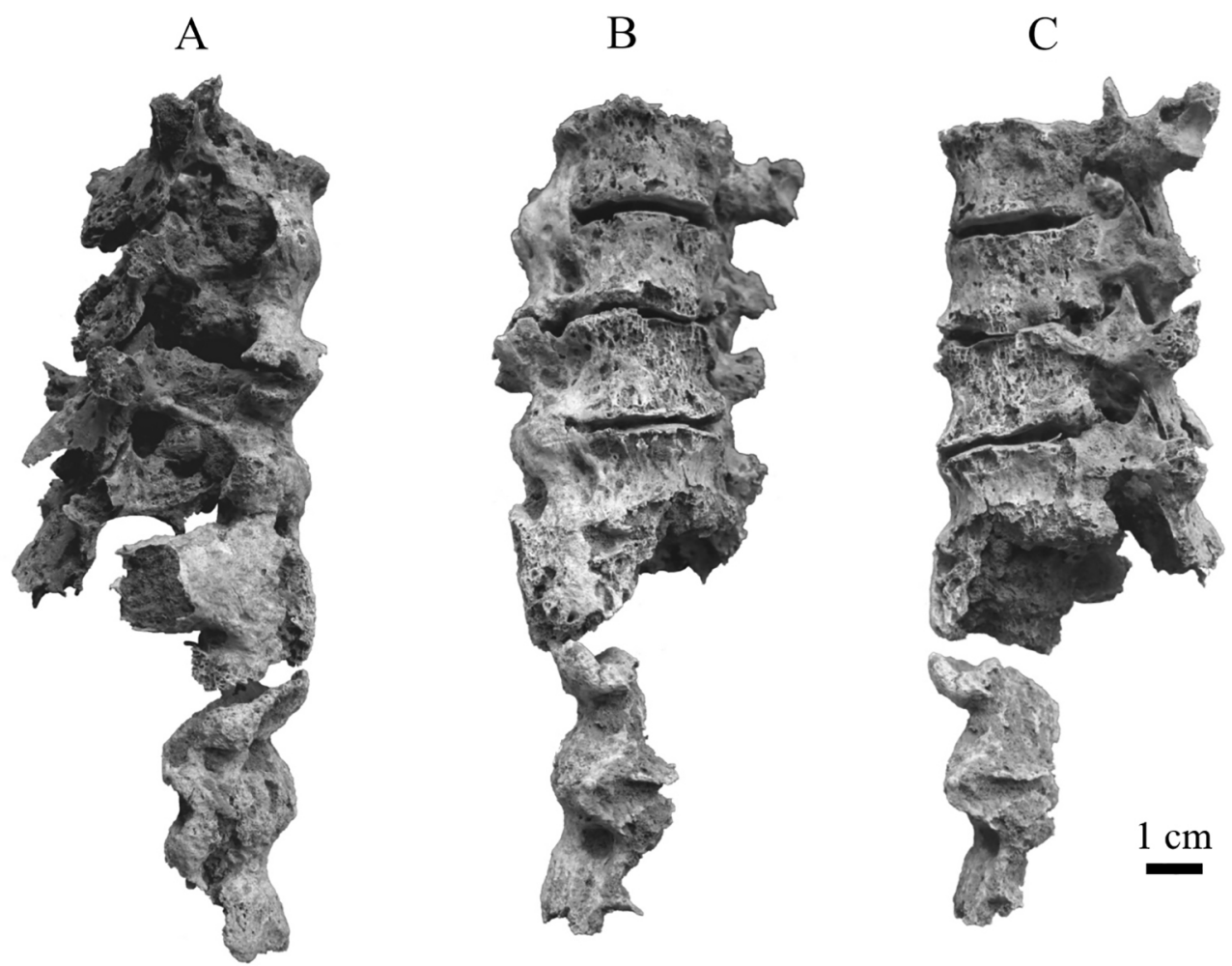

Fig. 9 Sept vertèbres thoraciques de l'individu de la tombe 11 de Kindoki. A. Vue latérale droite. B. Vue antérieure. C. Vue latérale gauche / Seven thoracic vertebrae of the individual from grave $n^{\circ} 11$ at Kindoki. A. Right side view. B. Anterior view. C. Left side view

on ne dispose de pratiquement aucune information sur l'alimentation et la santé de ces aristocrates du royaume Kongo. Les comparaisons réalisées avec d'autres populations d'Afrique centrale (voir ci-dessous) sont, à ce stade, exploratoires. Elles ont pour but de mettre en évidence si l'échantillon privilégié de Kindoki se démarque des autres populations de la région.

Les deux statures féminines de Kindoki qui avoisinent $1 \mathrm{~m}$ 55-1 m 60 sont comparables à celles des populations actuelles d'Afrique centrale (à l'exception des Pygmées) comme les Bakiga vivant au sud-ouest de l'Ouganda et au nord du Rwanda (moyenne féminine $=155,1 \mathrm{~cm}$ ) [58], les Lese de RDC (153 cm), les Bagandu et Issongo de la République centrafricaine $(154 \mathrm{~cm})$ [59]. La stature masculine qui est de l'ordre de $1 \mathrm{~m} 60$ est par contre inférieure à celle des populations actuelles d'Afrique centrale comme les Bakiga (moyenne masculine $=165,4 \mathrm{~cm}$ ) et les Luba $(165,7 \mathrm{~cm})$ [60] ainsi qu'à celle des populations de la RDC datées du $\mathrm{VIIII}^{\mathrm{e}}$ au XV $\mathrm{v}^{\mathrm{e}}$ siècle comme les Sanga $(165,7 \mathrm{~cm})$ et les Katoto $(165,8 \mathrm{~cm})$ [61]. Elle est néanmoins similaire à celle des Lese $(161 \mathrm{~cm})$ et des Bagandu et Issongo $(159 \mathrm{~cm})$ [62].

Les fusions vertébrales principalement localisées du côté droit chez les individus féminins des tombes 8 et 11 sont évocatrices de DISH ou de maladie hyperostosique [29] (pp. 47-54 et 62-64). Ses manifestations rachidiennes consistent en une ossification du ligament vertébral commun antérieur. Au niveau thoracique, celle-ci est limitée au côté droit suite aux pulsations de l'aorte [65]. Le diagnostic de DISH repose sur la présence d'une coulée osseuse impliquant au moins quatre vertèbres thoraciques consécutives [63]. Il est donc confirmé pour l'individu 11, mais reste probable pour l'individu 8 . Les fusions costovertébrale et sternocostale du sujet 8 ne font pas partie des signes attribués habituellement au DISH. Elles sont par contre associées à des spondylarthropathies comme la spondylarthrite ankylosante [66]. Le DISH est également caractérisé par des manifestations extrarachidiennes comme des ossifications de diverses enthèses (au niveau des talons, des coudes, des chevilles, des genoux et des épaules) [64,67], mais le mauvais état de conservation des individus ne nous permet pas de les observer. Actuellement, cette affection touche généralement les individus masculins (sex-ratio : 2/1) de plus de 50 ans et est associée à une alimentation riche, à l'obésité et au diabète de type II [68]. Il est donc envisageable que les deux femmes inhumées dans ce cimetière aient eu, de par leur statut social, accès à une alimentation très riche et qu'elles étaient en surpoids. Des cas archéologiques de DISH ont déjà été associés à un statut social élevé [69-73]. Les données actuelles montrent qu'environ $4 \%$ des Noirs africains présentent cette pathologie [65]. Des cas archéologiques de DISH ont été 
décrits en Afrique du Nord [74,75] et au Botswana [76], mais encore jamais en Afrique centrale.

Le LSAMAT serait dû à la mastication d'aliments très abrasifs comme les tubercules [44,45]. Irish et Turner [77] l'ont observé chez des Sénégalais d'époque contemporaine ( $\mathrm{XIX}^{\mathrm{e}}$ siècle) et l'ont mis en relation avec la consommation de manioc. Cela pourrait avoir aussi été le cas à Kindoki, car cette plante originaire d'Amérique du Sud fut introduite dans le bassin du Congo par les Portugais au XvI ${ }^{\mathrm{e}}$ siècle [78] (pp. 60-87).

Deux des dix individus de Kindoki présentaient des caries dentaires, et le taux de dents cariées s'élève à 1,6\%. Il n'existe malheureusement pas de données comparatives pour l'Afrique centrale à l'époque concernée (fin XVII -début $\mathrm{XIX}^{\mathrm{e}}$ siècle). On dispose toutefois de données pour des échantillons plus anciens et plus récents. Les fréquences sont toutes plus élevées qu'à Kindoki. Ainsi, Gutierrez et Valentin ont relevé 6,3\% de dents cariées chez des Angolais de statut social élevé décédés entre le $\mathrm{XVI}^{\mathrm{e}}$ et le $\mathrm{XVII}^{\mathrm{e}}$ siècle [79]. L'étude menée par Dlamini et al. sur les Sanga et les Katoto, des agriculteurs du Katanga (province la plus méridionale de la RDC) datés du VIII ${ }^{\mathrm{e}}$ au $\mathrm{xv}^{\mathrm{e}}$ siècle, a montré des taux de carie respectifs de 17,1 et 3,9\% [80]. Brabant, qui a étudié des crânes hutus du Rwanda datés du début du $\mathrm{xx}^{\mathrm{e}}$ siècle, mentionne une fréquence de dents cariées de $4,6 \%$ qui affectaient $20 \%$ des individus (comme à Kindoki) [81]. Walker et Hewlett ont relevé à la fin $\mathrm{du} \mathrm{xx}^{\mathrm{e}}$ siècle des fréquences de dents cariées de 5,2 à $6 \%$ chez les Pygmées chasseurscueilleurs et de $8,1 \%$ chez les Bantous agriculteurs [82]. Ils ont aussi observé que les Pygmées de statut social plus élevé présentaient moins de dents cariées que les autres individus $(5,7$ contre $7,7 \%)$.

Trois individus de Kindoki présentent des dépôts de tartre mais, étant donné le mauvais état de préservation, certains autres individus pourraient l'avoir perdu post mortem. Des squelettes inhumés dans des conditions plus favorables (en grotte) présentent des prévalences plus élevées comme les Hutus du Rwanda, dont la moitié montrait des dépôts de tartre [81].

Chez les trois individus les mieux conservés de Kindoki, il a été possible de mettre en évidence un mauvais état de santé buccodentaire via la présence de maladie parodontale et de lésions périapicales. Les parodontites sont liées à des facteurs génétiques, hormonaux, systémiques (diabètes de types I et II et ostéoporose), locaux (problèmes occlusaux et présence de tartre), sociaux et comportementaux (tabagisme, stress et mauvaise hygiène buccodentaire) [83]. Il est donc probable qu'à Kindoki le tabagisme, la présence de tartre et peut-être du diabète (souvent associé au DISH) aient accru le risque de développer une maladie parodontale. Près de la moitié des Hutus du Rwanda présentait des signes de cette affection [81]. Les atteintes périapicales infectieuses peuvent faire suite à une carie, à un traumatisme dentaire, à une usure importante ou à la propagation depuis un foyer infectieux voisin (gingivite, parodontite, sinusite) [42] (pp. 143-146). À Kindoki, aucune carie ne semble à l'origine de lésions périapicales. Il est donc possible que ces dernières soient plutôt liées à la parodontopathie. Ces lésions périapicales étaient aussi présentes dans d'autres populations archéologiques d'Afrique centrale, comme les Hutus du Rwanda (16,8 \%) [81].

Les hypercémentoses sont généralement causées par des surcharges mécaniques ou une inflammation périapicale [42] (pp. 255-256). Les deux types de facteurs (consommation d'aliments durs et inflammation de l'os alvéolaire) pourraient avoir joué un rôle à Kindoki.

Seul un individu présente de l'hypoplasie de l'émail dentaire, un marqueur de maladie et/ou une malnutrition pendant la croissance. Cette faible prévalence pourrait être liée à leur statut social privilégié, comme cela a été mis en évidence dans d'autres échantillons anciens $[84,85]$ ou récents $[86,87]$.

\section{Conclusion}

Les fouilles réalisées sur la colline de Kindoki ont permis la découverte d'un petit cimetière qui renfermait au moins 11 tombes d'aristocrates datées $\mathrm{du} \mathrm{XVII}^{\mathrm{e}}$ au $\mathrm{XIX}^{\mathrm{e}}$ siècle. Comme dans de nombreux autres sites de plein air localisés en climat tropical humide, les restes humains sont très mal conservés. Deux tombes étaient même dépourvues de restes humains. Les études anthropologique et archéologique ont néanmoins révélé la présence de huit hommes et de deux femmes.

Au moins deux de ces hommes étaient des fumeurs de pipe. Une femme était atteinte de DISH, et il est probable que la seconde souffrait aussi de cette maladie. Cette pathologie, qui n'avait pas encore été décrite chez des populations archéologiques d'Afrique centrale, est probablement en relation avec leur statut élevé. Les faibles taux de carie dentaire et d'hypoplasie de l'émail dentaire pourraient, eux aussi, être liés à cette position sociale favorisée. Leur stature, par contre, n'est pas élevée et est comparable (voire inférieure) à celle d'individus vivant actuellement ou il y a quelques centaines d'années en Afrique centrale.

Le tabagisme et le déséquilibre glycémique pourraient être à l'origine de leur mauvais état de santé buccodentaire.

L'étude des particularités anatomiques osseuses n'a pas permis d'identifier d'éventuels liens de parenté au sein des individus inhumés dans ce cimetière. Une étude des caractères discrets dentaires et des analyses d'ADN pourraient apporter des éléments supplémentaires à cette problématique.

L'usure particulière des incisives supérieures (LSAMAT) indique la consommation d'aliments abrasifs comme le manioc. L'étude des microfossiles végétaux emprisonnés 
dans le tartre dentaire et des analyses isotopiques du collagène osseux sont en cours. Elles pourraient permettre de confirmer cette hypothèse et d'apporter de nouvelles informations sur le régime alimentaire de ces dignitaires du royaume Kongo.

Remerciements Le projet KongoKing a été financé par le Conseil européen de la recherche (European Research Council Starting Grant No. 284126). Nous adressons nos remerciements à Hélène Déom (UCL/University of Sheffield),
Mélodie Ducastelle (ULB), Camille Delmarcelle (UCL) et Nathan Van Kerkhoven (ULB) pour leur aide lors du nettoyage et de l'étude des squelettes. Nous remercions AnneMarie Wittek (ADIA) pour la réalisation des dessins et Thierry Leduc (IRSNB) pour les analyses chimiques des restes osseux. Les commentaires constructifs des deux relecteurs anonymes ont permis d'améliorer cet article.

Liens d'intérêts : les auteurs déclarent ne pas avoir de lien d'intérêt. 


\section{Annexe A}

Représentation schématique du squelette de la tombe 8 de Kindoki. Les couleurs des restes osseux y sont figurées : les os de teinte «normale » sont en gris (dessin : M. Coutureau [INRAP] et A.-M. Wittek [ADIA]) / Diagram of the skeleton from grave $\mathrm{n}^{\circ \circ} 8$ at Kindoki, indicating the colours of the bone remains: the bones of "normal" colour are in grey (drawing : M. Coutureau [INRAP] and A.-M. Wittek [ADIA])

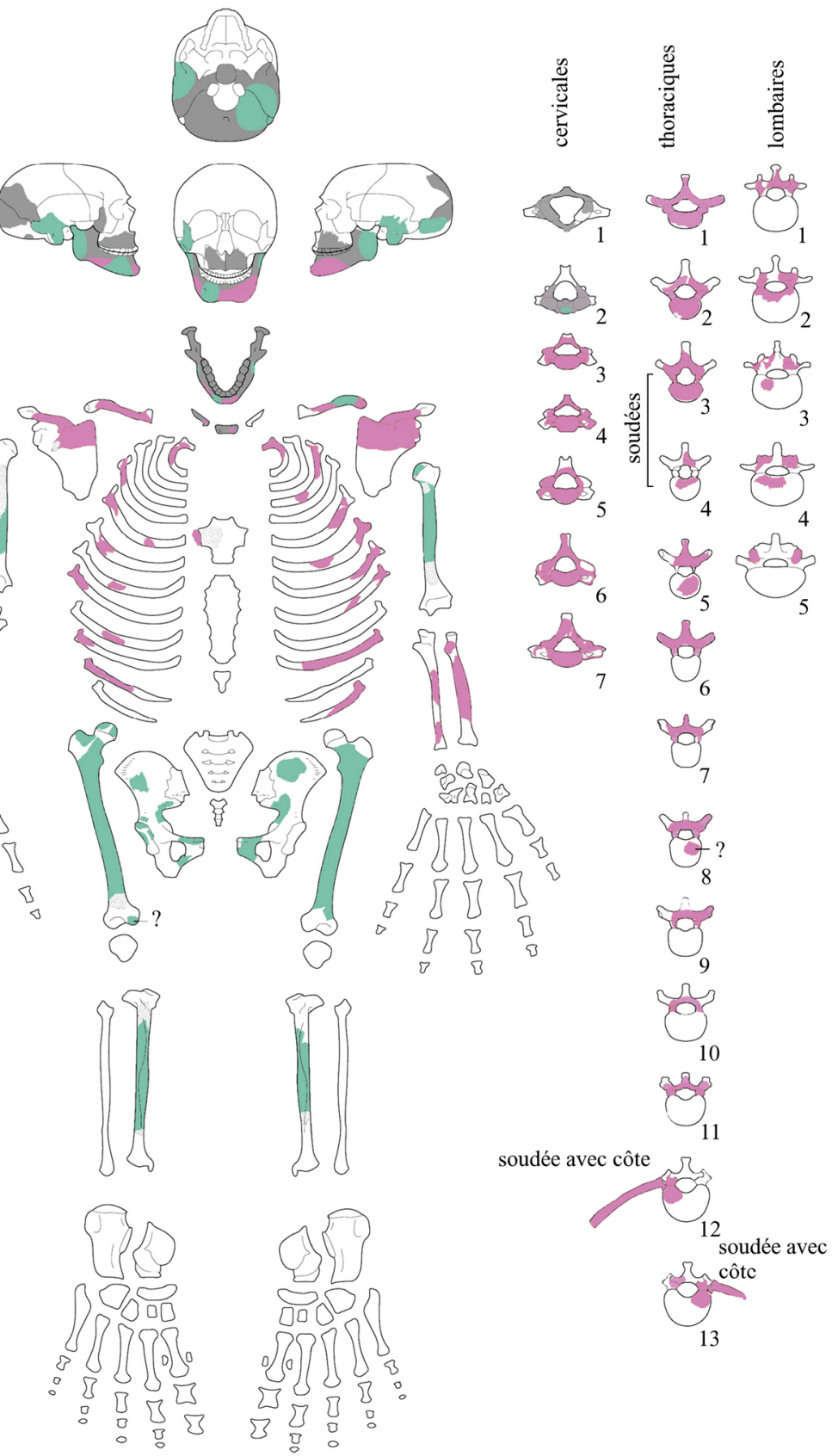




\section{Annexe B}

Représentation schématique du squelette de la tombe 9 de Kindoki. Les couleurs des restes osseux y sont figurées : les os de teinte « normale » sont en gris (dessin : M. Coutureau [INRAP] et A.-M. Wittek [ADIA]) / Diagram of the skeleton from grave $\mathrm{n}^{\circ} \mathrm{9}$ at Kindoki, indicating the colours of the bone remains: the bones of "normal" colour are in grey (drawing : M. Coutureau [INRAP] and A.-M. Wittek [ADIA])

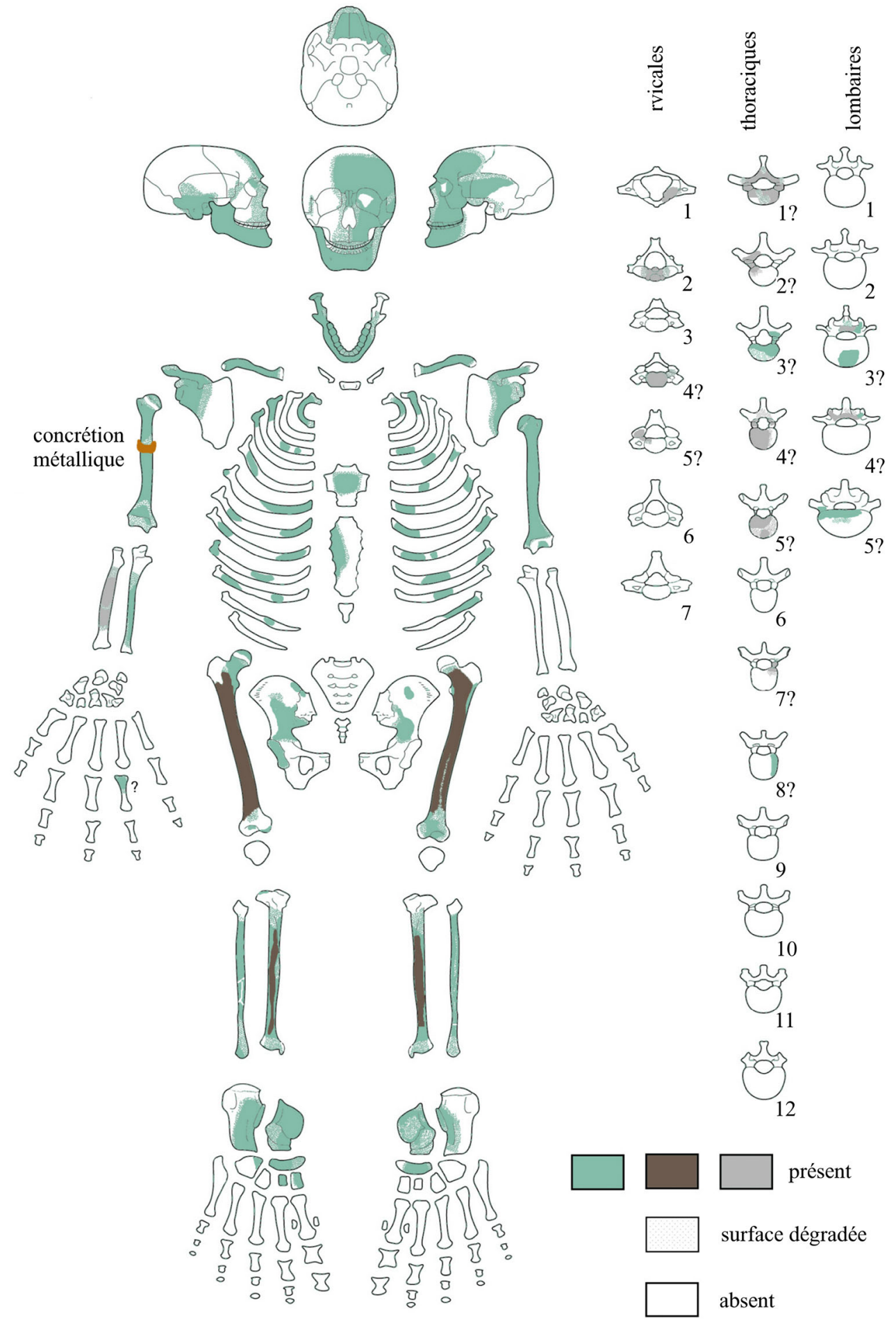




\section{Annexe C}

Représentation schématique du squelette de la tombe 11 de Kindoki. Les couleurs des restes osseux y sont figurées : les os de teinte «normale » sont en gris (dessin : M. Coutureau [INRAP] et A.-M. Wittek [ADIA]) / Diagram of the skeleton from grave $\mathrm{n}^{\circ} \mathrm{1} 11$ at Kindoki, indicating the colours of the bone remains: the bones of "normal" colour are in grey (drawing : M. Coutureau [INRAP] and A.-M. Wittek [ADIA])

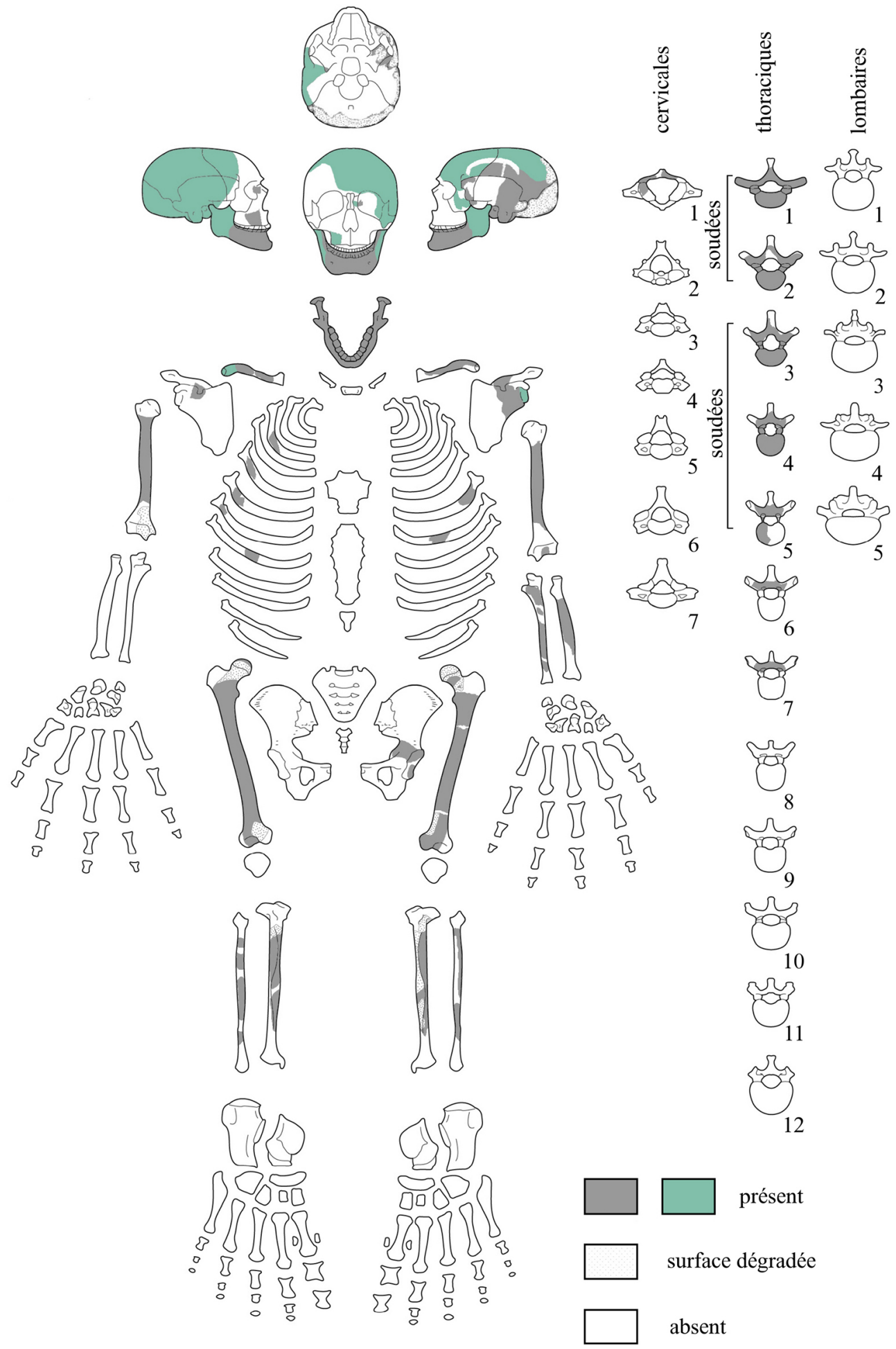




\section{Références}

1. Bostoen K, KongoKing. http://research.flw.ugent.be/en/projects/ kongoking

2. Clist B, Cranshof E, de Schryver GM, et al (2015) The elusive archaeology of Kongo urbanism: the case of Kindoki, Mbanza Nsundi (Lower Congo, DRC). Afr Archaeol Rev 32:369-412

3. Thornton J (2001) The origins and early history of the Kingdom of Kongo. Int J Afr Hist Stud 34:89-120

4. Clist B, Cranshof E, de Schryver GM, et al (2015), AfricanEuropean contacts in the Kongo Kingdom (sixteenth-eighteenth centuries): new archaeological insights from Ngongo Mbata (Lower Congo, DRC). Int J Hist Archaeol 19:464-501

5. Esteves E (1989) Mbanza Kongo, ville archéologique. Nsi 6:159-64

6. Souindoula S (1992) Mbanza Soyo: pesquisas arqueolôgicas. Leba 7:277-82

7. Vandenhoute J (1972-1973) De begraafplaats van Ngongo-Mbata (Neder-Zaire). Opgravningsverslag en historische situering. Thèse de master non publiée, Rijksuniversiteit Gent, Gand (Belgique)

8. Ladmirant H, Annaert A (1970) Carte géologique du Congo. Feuille Inkisi S.6/15. Institut géographique militaire de Belgique, Bruxelles

9. Bultot F (1950) Carte des régions climatiques du Congo belge établie d'après les critères de Köppen. INEAC, Publications du Bureau climatique 9, Bruxelles, $15 \mathrm{p}$

10. Wamuini Lunkayilakio S (2010). Ichtyofaune de l'Inkisi (BasCongo / RDC) : diversité et écologie. Thèse de doctorat en sciences non publiée de l'Université de Liège (Belgique), $351 \mathrm{p}$

11. de Maret P (2002) Urban origins in central Africa - The case of Kongo. In: Sinclair PJJ (ed) The development of urbanism from a global perspective. University of Uppsala, Uppsala, pp 1-15

12. Verhaeghe C, Clist B, Fontaine C, et al (2014) Shell and glass beads from the tombs of Kindoki, Mbanza Nsundi, Lower Congo. Beads 33:22-33

13. Bontinck F, Castro Segovia J (1972) Histoire du royaume du Congo (c. 1624). Études d'Histoire africaine IV, Éditions Nauwelaers, Beauvechain (Belgique), $145 \mathrm{p}$

14. Thornton J (2013) Afro-christian syncretism in the Kingdom of Kongo. J Afr Hist 54:53-77

15. Dutour O (1989) Hommes fossiles du Sahara : peuplements holocènes du Mali septentrional. Éditions du CNRS, Paris, $342 \mathrm{p}$

16. McKinley JI (2004) Compiling a skeletal inventory: disarticulated and co-mingled remains. In: Brickley M, McKinley JI (eds) Guidelines to the standards for recording human remains. BABAO and Institute of Field Archaeologists, Southampton (UK), IFA Paper No. 7, pp 14-7

17. Ferembach D, Schwidetzky I, Stloukal M (1979) Recommandations pour déterminer l'âge et le sexe sur le squelette. Bull Mem Soc Anthropol Paris 6:7-45

18. Krogman WM, İscan MY (1986) The human skeleton in forensic medicine. 2nd edition. CC Thomas, Springfield, $551 \mathrm{p}$

19. Franklin D, O'Higgins P, Oxnard CE, et al (2006) Determination of sex in South African Blacks by discriminant function analysis of mandibular linear dimensions: a preliminary investigation using the Zulu local population. Forensic Sci Med Pathol $2: 263-8$

20. Dibennardo K, Taylor JV (1982) Classification and misclassification in sexing the Black femur by discriminant function analysis. Am J Phys Anthropol 58:145-51

21. İşcan MY, Miller-Shaivitz P (1984) Determination of sex from the tibia. Am J Phys Anthropol 64:53-7
22. Macaluso PJ Jr (2010) Sex discrimination potential of permanent maxillary molar cusp diameters. J Forensic Odontostomatol 28:22-31

23. Orban R, Semal P, Molleson T (1989) La nécropole médiévale de Coxyde (Belgique) : propos sur la détermination du sexe. Anthropol Prehist 100:57-70

24. Lovejoy CO (1985) Dental wear in the Libben population: its functional pattern and role in the determination of adult skeletal age at death. Am J Phys Anthropol 68:47-56

25. Scheuer L, Black S (2000) Developmental juvenile osteology. Academic Press, San Diego, $587 \mathrm{p}$

26. Trotter M, Gleser G (1952) Estimation of stature from long bones of American Whites and Negroes. Am J Phys Anthropol 10:463514

27. Steele DG, McKern TW (1969) A method for assessment of maximum long bone length and living stature from fragmentary long bones. Am J Phys Anthropol 31:215-27

28. Aufderheide AC, Rodríguez-Martín C (1998) The Cambridge encyclopedia of human paleopathology. Cambridge University Press, Cambridge, $478 \mathrm{p}$

29. Rogers J, Waldron T (1995) A field guide to joint disease in archaeology. John Wiley \& Sons, Chichester, $119 \mathrm{p}$

30. Hillson S (1996) Dental anthropology. Cambridge University Press, Cambridge, $373 \mathrm{p}$

31. Brothwell, DR (1963) Digging up bones. The excavation, treatment and study of human skeletal remains. British Museum, Londres, $194 \mathrm{p}$

32. Olivier G (1960) Pratique anthropologique. Vigot frères, Paris, $299 \mathrm{p}$

33. Capasso L, Kennedy KAR, Wilczak CA (1999) Atlas of occupational markers on human remains. Atto, Teramo (Italie), $183 \mathrm{p}$

34. Dupras TL, Schultz JJ (2013) Taphonomic bone staining and color changes in forensic contexts. In: Pokines J, Symes SA (eds) Manual of forensic taphonomy. CRC Press, Boca Raton (USA), pp 315-40

35. Clist B, de Maret P, Bostoen K (eds) Archéologie des provinces septentrionales du royaume Kongo. Oxford, Archaeopress [in press]

36. Stewart TD (1957) Rate of development of vertebral hypertrophic arthritis and its utility in age estimation. Am J Phys Anthropol 15: 433

37. Simon L, Claustre J, Blotman F (1984) Abrégé de rhumatologie. $4^{\mathrm{e}}$ éd. Masson, Paris, $592 \mathrm{p}$

38. Mikkelsen WM, Duff IF, Dodge HJ (1970) Age-sex specific prevalence of radiographic abnormalities of the joints of the hands, wrists and cervical spine of adult residents of Tecumseh, Michigan, Community Health Study area, 1962-1965. J Chronic Dis 23:151-9

39. Metress JF, Conway T (1975) Standardized system for recording dental caries in prehistoric skeletons. J Dent Res 54:908

40. Duyninh T, Jame O, Bousquet P, et al (2005) Classification des maladies parodontales. EMC-Odontologie 1:58-66

41. Brabant H, Sahly A (1962) La paléostomatologie en Belgique et en France : essai de synthèse de recherches personnelles. Acta Stomatol Belg 59:286-355

42. Piette E, Goldberg M (2001) La dent normale et pathologique. De Boeck Université, Bruxelles, 392 p

43. Dias G, Tayles N (1997) "Abscess cavity" — a misnomer. Int J Osteoarchaeol 7:548-54

44. Becker A, Karnei-R'em RM (1992) The effects of infraocclusion: Part 1. Tilting of the adjacent teeth and local space loss. Am J Orthod Dentofacial Orthop 102:256-64

45. Turner CG 2nd, Machado LM (1983) A new dental wear pattern and evidence for high carbohydrate consumption in a Brazilian archaic skeletal population. Am J Phys Anthropol 61:125-30 
46. Dent BB, Forbes SL, Stuart BH (2004) Review of human decomposition processes in soil. Environ Geol 45:576-85

47. Hassett B (2006) Torus mandibularis: etiology and bioarcheological utility. Dental Anthropology 19:1-14

48. Hauser G, De Stefano GF (eds) (1989) Epigenetic variants of the human skull. Schweizerbart, Stuttgart, $301 \mathrm{p}$

49. Chapman T, Sholukha V, Semal P, et al (2015) Femoral curvature variability in modern humans using three-dimensional quadric surface fitting. Surg Radiol Anat 37:1169-77

50. Reginato A, Roques JC, Pommereau X, et al (1987) Le torus mandibularis. Bull Mem Soc Anthropol Paris 4:25-32

51. Corruccini RS (1974) An examination of the meaning of cranial discrete traits for human skeletal biological studies. Am J Phys Anthropol 40:425-45

52. Ajmani ML, Mittal RK, Jain SP (1983) Incidence of the metopic suture in adult Nigerian skulls. J Anat 137:177-83

53. Olivier G (1965) Anatomie anthropologique. Vigot frères, Paris, $488 \mathrm{p}$

54. Walensky NA (1965) A study of anterior femoral curvature in man. Anat Rec 151:559-70

55. Crubézy É, Telmon N, Sevin A, et al (1999) Microévolution d'une population historique. Étude des caractères discrets de la population de Missiminia (Soudan, $\mathrm{III}^{\mathrm{e}}-\mathrm{VI}^{\mathrm{e}}$ siècle). Bull Mem Soc Anthropol Paris 11:1-213

56. Scott GR, Turner II CG (1988) Dental anthropology. Annu Rev Anthropol 17:99-126

57. Jones A (1983) Samuel Brun's voyages of 1611-20. In: Jones A (ed) German sources for West-African history, 1599-1669. Wiesbaden, Franz Steiner, pp 60-1

58. Perry GH, Foll M, Grenier JC, et al (2014) Adaptive, convergent origins of the pygmy phenotype in African rainforest huntergatherers. Proc Natl Acad Sci 111: E3596-E603

59. Eveleth PB, Tanner JM (1990) Worldwide variation in human growth. Cambridge University Press, Cambridge, 2nd edition, $397 \mathrm{p}$

60. Hiernaux J (1964) Luba du Katanga et Luba du Kasai (Congo) ; comparaison de deux populations de même origine. Bull Mem Soc Anthropol Paris 6:611-22

61. Hiernaux J, Plantier M, De Buyst J (1992) Étude ostéométrique des restes humains de Sanga et Katoto (âge du fer, Zaïre). Anthropol Prehist 103:9-44

62. Holgate RL, Steyn M (2016) Diffuse idiopathic skeletal hyperostosis: diagnostic, clinical and paleopathological considerations. Clin Anat 29:870-7

63. Crubézy É, Crubézy-Ibáñez E (1993) Évaluation sur une série de squelettes de critères diagnostiques de la maladie hyperostosique. Implications épidémiologiques. Rev Rhum 60:586-90

64. Resnick D, Niwayama G (1976) Radiographic and pathologic features of spinal involvement in diffuse idiopathic skeletal hyperostosis (DISH). Radiology 119:559-68

65. Utsinger PD (1985) Diffuse idiopathic skeletal hyperostosis. Clin Rheum Dis 11:325-51

66. Olivieri I, D’Angelo S, Palazzi C, et al (2009) Diffuse idiopathic skeletal hyperostosis: differentiation from ankylosing spondylitis. Curr Rheumatol Rep 11:321-8

67. Mader R, Sarzi-Puttini P, Atzeni F, et al (2009) Extraspinal manifestations of diffuse idiopathic skeletal hyperostosis. Rheumatology 48:1478-81

68. Burner TW, Rosenthal AK (2009) Diabetes and rheumatic diseases. Curr Opin Rheumatol 21:50-4
69. Rogers J, Waldron T (2001) DISH and the monastic way of life. Int J Osteoarchaeol 11:357-65

70. Jankauskas R (2003) The incidence of diffuse idiopathic skeletal hyperostosis and social status correlations in Lithuanian skeletal materials. Int J Osteoarchaeol 13:289-93

71. Giuffra V, Giusiani S, Fornaciari A, et al (2010) Diffuse idiopathic skeletal hyperostosis in the Medici, Grand Dukes of Florence (XVI century). Eur Spine J 19:S103-S7

72. Blondiaux J, Alduc-Le Bagousse A, Demondion X, et al (2007) Maladie hyperostosique et maladie goutteuse, une diathèse familiale en Normandie : Thaon, Calvados. Bull Mem Soc Anthropol Paris 19:7-20

73. Kacki S, Villotte S (2006) Maladie hyperostosique et mode de vie : intérêt d'une démarche bioarchéologique. Exemple du cimetière du couvent des Sœurs Grises de Beauvais (Oise), $\mathrm{Xv}^{\mathrm{e}}-$ $\mathrm{XVIII}^{\mathrm{e}}$ siècles. Bull Mem Soc Anthropol Paris 18:55-64

74. Dupras TL, Williams LJ, Willems H, et al (2010) Pathological skeletal remains from ancient Egypt: the earliest case of diabetes mellitus? Practical Diabetes Int 27:358-63a

75. Saleem SN, Hawass Z (2014) Ankylosing spondylitis or diffuse idiopathic skeletal hyperostosis in royal Egyptian mummies of 18th-20th Dynasties? CT and archaeology studies. Arthritis Rheumatol 66:3311-6

76. Mosothwane MN, Steyn M (2009) In sickness or in health? Assessment of Early Iron Age human skeletons from Toutswe sites, east central Botswana. Int J Osteoarchaeol 19:66-77

77. Irish JD, Turner CG 2nd (1997) First evidence of LSAMAT in non-native Americans: historic Senegalese from West Africa. Am J Phyl Anthropol 102:141-6

78. Jones W (1959) Manioc in Africa. Stanford University Press, Stanford, $315 \mathrm{p}$

79. Gutierrez M, Valentin F (1995) Archéologie et anthropologie des tumulus de Kapanda (Angola). J Africanistes 65:145-69

80. Dlamini N, Morris AG, Sealy J (2016) Carbon isotopes and dental caries as evidence for regional variation in the diets of early farming communities from Katanga, Democratic Republic of the Congo. J Afr Archaeol 14:135-53

81. Brabant H (1963) Observations anthropologiques et odontologiques sur les dents des Hutus du Rwanda. Musée royal de 1'Afrique centrale, Tervuren (Belgique), Annales, séries in- $8^{\circ}$. Sciences humaines, $\mathrm{n}^{\circ} 47,30 \mathrm{p}$

82. Walker PL, Hewlett BS (1990) Dental health, diet and social status among Central African foragers and farmers. Am Anthropol 92:383-98

83. Nunn ME (2003) Understanding the etiology of periodontitis: an overview of periodontal risk factors. Periodontol 2000 32:11-23

84. Nakayama N (2016) The relationship between linear enamel hypoplasia and social status in 18th to 19th Century Edo, Japan. Int J Osteoarchaeol 26:1034-44

85. Miszkiewicz JJ (2012) Linear enamel hypoplasia and ageat-death at Medieval (11th-16th Centuries) St. Gregory's Priory and Cemetery, Canterbury, UK. Int J Osteoarchaeol 25:79-87

86. Skinner MF, Hung JTW (1989) Social and biological correlates of localized enamel hypoplasia of the human deciduous canine tooth. Am J Phyl Anthropol 79:159-75

87. Lukacs JR (1991) Localized enamel hypoplasia of human deciduous canine teeth: prevalence and pattern of expression in rural Pakistan. Hum Biol 63:513-22 